\title{
QUASICONFORMAL AND GEODESIC TREES
}

\author{
MARIO BONK AND DANIEL MEYER
}

\begin{abstract}
A quasiconformal tree is a metric tree that is doubling and of bounded turning. We prove that every quasiconformal tree is quasisymmetrically equivalent to a geodesic tree with Hausdorff dimension arbitrarily close to 1 .
\end{abstract}

\section{Contents}

1. Introduction

1.1. Notation 6

2. Auxiliary facts 7

3. Sun and shadow 13

4. Good double points 18

5. Subdividing the tree 21

6. Weights and main vertices of tiles 33

7. Construction of the geodesic metric 38

8. Quasisymmetry 46

9. Lowering the Hausdorff dimension 48

10. Remarks and open problems 49

References 50

\section{INTRODUCTION}

An important question in geometric analysis is whether a given metric space (belonging to some class of spaces) is geometrically equivalent to a model space in a natural way. Many results in mathematics can be seen from this perspective (such as the existence of isothermal or conformal coordinates on surfaces or the Riemann mapping theorem). For general metric spaces there are various ways to interpret geometric equivalence: up to isometric or up to bi-Lipschitz equivalence, for

Date: June 11, 2020.

2010 Mathematics Subject Classification. Primary 30L10; Secondary 51F99.

Key words and phrases. Quasiconformal tree, geodesic tree, quasisymmetry, doubling metric spaces, bounded turning, conformal dimension. 
example. In the present paper the relevant notion of geometric equivalence is based on a class of homeomorphisms that are close to conformal or quasiconformal maps in a classical complex-analytic context, namely quasisymmetries.

By definition, a homeomorphism $f: X \rightarrow Y$ between metric spaces $\left(X, d_{X}\right)$ and $\left(Y, d_{Y}\right)$ is said to be quasisymmetric or a quasisymmetry, if there exists a homeomorphism $\eta:[0, \infty) \rightarrow[0, \infty)$ (playing the role of a control function for distortion) such that

$$
\frac{d_{Y}(f(x), f(y))}{d_{Y}(f(x), f(z))} \leq \eta\left(\frac{d_{X}(x, y)}{d_{X}(x, z)}\right)
$$

for all distinct points $x, y, z \in X$. The composition of two quasisymmetries (when defined) and the inverse of a quasisymmetry are quasisymmetric. So if we call two metric spaces $X$ and $Y$ quasisymmetrically equivalent if there exists a quasisymmetry $f: X \rightarrow Y$, then we have a notion of geometric equivalence for metric spaces. Since every biLipschitz homeomorphism is a quasisymmetry, this is a weaker, and hence more flexible, notion than bi-Lipschitz (or even isometric) equivalence (for more background and related discussions see [BM17, Section 4.1] and [He01, Chapters 10-12]).

The quasisymmetric uniformization problem (see [Bo06]) asks for natural conditions when a given metric space $X$ from a class of spaces is quasisymmetrically equivalent to some model space $Y$. This problem is relevant in various contexts. For example, the Kapovich-Kleiner conjecture in geometric group theory (see [KK00, Conjecture 6]) amounts to the problem of showing that every Sierpiński carpet arising as the boundary of a Gromov hyperbolic group is quasisymmetrically equivalent to a "round" Sierpiński carpet (see Bo11 for a related discussion).

The prototypical instance of a quasisymmetric uniformization result is the characterization by Tukia and Väisälä of metric spaces quasisymmetrically equivalent to the unit interval $[0,1]$. In order to formulate their theorem we need two definitions.

We say that a metric space $(X, d)$ is of bounded turning if there exists a constant $K \geq 1$ such that for all $x, y \in X$ there exists a compact connected set $E \subset X$ with $x, y \in E$ and

$$
\operatorname{diam}(E) \leq K d(x, y)
$$

In this case, we say that $(X, d)$ is of $K$-bounded turning.

A metric space $(X, d)$ is doubling if there exists a constant $N \in \mathbb{N}$ (the doubling constant of $X$ ) such that each ball in $X$ of radius $R>0$ can be covered by $N$ (or fewer) balls of radius $R / 2$. 
Tukia and Väisälä showed that a metric space $J$ homeomorphic to $[0,1]$ is quasisymmetrically equivalent to $[0,1]$ if and only if it is doubling and of bounded turning (see [TV80]). In other words, one can "straighten out" the arc $J$ (which may well have Hausdorff dimension $>1$ ) to the interval $[0,1]$ by a quasisymmetry.

In the present paper, we study the quasisymmetric uniformization problem for metric trees. By definition, a (metric) tree is a compact, connected, and locally connected metric space $(\mathbf{T}, d)$ that contains at least two distinct points and has the following property: if $x, y \in \mathbf{T}$, then there exists a unique arc in $\mathbf{T}$ with endpoints $x$ and $y$. This arc is denoted by $[x, y]$. We allow $x=y$ here, in which case we consider $[x, y]=\{x\}$ as a degenerate arc.

The underlying topological space of a tree is often called a dendrite in the literature. Since we are mostly interested in metric properties and want to emphasize this metric aspect, we prefer the name tree for these objects. Motivated by the Tukia-Väisälä result and the connection with quasiconformal geometry, we introduce the following terminology.

Definition 1.1. A metric tree is quasiconformal if it is doubling and of bounded turning.

In the following, we usually call a quasiconformal tree a qc-tree for brevity.

Trees appear in many contexts in mathematics, for example as Julia sets of polynomials. The Julia set $\mathcal{J}(P)$ of the polynomial $P(z)=z^{2}+i$ is a tree (see [CG93, Example after Theorem V.4.2]). Actually, $\mathcal{J}(P) \subset$ $\mathbb{C}$ is a qc-tree if it is equipped with the ambient Euclidean metric on $\mathbb{C}$. Indeed, $\mathcal{J}(P)$ is of bounded turning as easily follows from the fact that $\mathbb{C} \backslash \mathcal{J}(P)$ is a John domain (see [CG93, Theorem VII.3.1]). Since every subset of a Euclidean space (such as the complex plane $\mathbb{C}$ ) is doubling, $\mathcal{J}(P)$ is doubling.

In analogy to the Tukia-Väisälä theorem one can raise the question whether all arcs in a qc-tree can be straightened out simultaneously by a quasisymmetry. For a precise formulation of this question the following concept is relevant.

A metric space $(X, d)$ is called geodesic if any two points $x, y \in X$ can be joined by a geodesic segment, i.e., by an arc $[x, y]$ with endpoints $x$ and $y$ whose length is equal to $d(x, y)$.

The following statement is the main result of this paper.

Theorem 1.2. Every quasiconformal tree is quasisymmetrically equivalent to a geodesic tree. 
Every arc that is doubling and of bounded turning is a qc-tree. This implies that Theorem 1.2 includes the Tukia-Väisälä theorem as a special case, and so can it be viewed as a generalization.

Various improvements and variants of Theorem 1.2 are conceivable. For example, one can ask whether additional assumptions yield quasisymmetric equivalence to a single specified space. We consider a question of this type in the follow-up paper [BM20, where it is shown that a qc-tree is quasisymmetrically equivalent to the continuum selfsimilar tree (as defined in [BT20]) if and only if it is trivalent and uniformly branching (see [BM20] for the relevant definitions).

Another natural question is "how small" we can make the geodesic tree $T$ that is the quasisymmetric image of the given qc-tree $\mathbf{T}$. If $\operatorname{dim}_{H} T$ denotes the Hausdorff dimension of $T$, then clearly $\operatorname{dim}_{H} T \geq 1$, because $T$ always contains a non-degenerate arc. We will show that $\operatorname{dim}_{H} T$ can actually be arbitrarily close to 1 and will establish the following improved version of Theorem 1.2 .

Theorem 1.3. If $\mathbf{T}$ is a quasiconconformal tree and $\alpha>1$, then $\mathbf{T}$ is quasisymmetrically equivalent to a geodesic tree $T$ with $\operatorname{dim}_{H} T \leq \alpha$.

In general, one cannot achieve $\operatorname{dim}_{H} T=1$ here. An example when this is not possible can be found in [BiT01] (see also Az15, Theorem 1.6] for a general related statement). If $\mathbf{T}$ is the continuum self-similar tree and $T$ is any tree that is quasisymmetrically equivalent to $\mathbf{T}$, then actually $\operatorname{dim}_{H} T>1$.

The conformal dimension $\operatorname{confdim}(X)$ of a metric space $X$ is defined as the infimum of all Hausdorff dimensions of metric spaces $Y$ that are quasisymmetrically equivalent to $X$. We refer to [MT10 for more background on this concept. Theorem 1.3 implies the following immediate consequence.

Corollary 1.4. If $\mathbf{T}$ is a quasiconformal tree, then $\operatorname{confdim}(\mathbf{T})=1$.

This last statement is not new, but was originally proved by Kinneberg [Kin17, Proposition 2.4].

We will now summarize the main ingredients for the proofs of Theorems 1.2 and 1.3 . The basic idea is to define a new geodesic metric $\varrho$ on the given qc-tree $(\mathbf{T}, d)$ so that the identity $\operatorname{map~id}_{\mathbf{T}}:(\mathbf{T}, d) \rightarrow(\mathbf{T}, \varrho)$ is a quasisymmetry. In order to define $\varrho$, we will carefully choose a sequence of decompositions $\mathbf{X}^{n}$ of $\mathbf{T}$ into subtrees. We call the elements $X^{n}$ in $\mathbf{X}^{n}$ tiles of level $n$ or $n$-tiles. To each $n$-tile $X^{n}$ we will assign a weight $w\left(X^{n}\right)$ by an inductive process on the level $n \in \mathbb{N}$. These weights can then be used to define a distance function $\varrho_{n}$ on $\mathbf{T}$ : one infimizes the total length with respect to this weight over chains of 
$n$-tiles from one point in $\mathbf{T}$ to another (see (6.1) and (7.1)). We will show that with our choices, the limit

$$
\varrho(x, y)=\lim _{n \rightarrow \infty} \varrho_{n}(x, y)
$$

exists for all $x, y \in \mathbf{T}$ (Lemma 7.3) and defines a geodesic metric on $\mathbf{T}$ (Lemma 7.6). We have $\operatorname{diam}_{\varrho}(X) \asymp w(X)$ for the $\varrho$-diameter of each tile $X$ (see Proposition 7.7 (i) . So in a sense the metric $\varrho$ is a "conformal" deformation of the original metric $d$ on $\mathbf{T}$ controlled by the weight $w(X)$ near each tile $X$. The fact that $\operatorname{id}_{\mathbf{T}}:(\mathbf{T}, d) \rightarrow(\mathbf{T}, \varrho)$ is a quasisymmetry can then easily be derived from geometric properties of tiles (see Lemma 8.2). Theorem 1.2 follows.

The choice of the weights and hence the construction of $\varrho$ involves a parameter $\epsilon_{0}>0$. We will see that if we choose $\epsilon_{0}$ close to 0 , then the Hausdorff dimension of $(\mathbf{T}, \varrho)$ is close to 1 . This immediately gives Theorem 1.3 .

The main difficulty in this general approach is how to define the decompositions $\mathbf{X}^{n}$. It is a natural idea to "cut" the tree $\mathbf{T}$ into subtrees by using auxiliary points. We will indeed follow this procedure by defining an ascending sequence of finite sets $\mathbf{V}^{1} \subset \mathbf{V}^{2} \subset \ldots$ that we use to cut $\mathbf{T}$. More precisely, the tiles of level $n$ are precisely the closures of the complementary components of $\mathbf{V}^{n}$, i.e., the closures of the components of $\mathbf{T} \backslash \mathbf{V}^{n}$. The construction of the sets $\mathbf{V}^{n}$ involves a (small) parameter $\delta \in(0,1)$. For each $n$-tile $X^{n}$ we will then have $\operatorname{diam}_{d}\left(X^{n}\right) \asymp \delta^{n}$. All of this looks natural and even straightforward, but there is a surprising subtlety here. Namely, one might expect that the $n$-vertices, i.e., the elements in $\mathbf{V}^{n}$ used for cutting the tree, should be branch points of $\mathbf{T}$ (points $b \in \mathbf{T}$ such that $\mathbf{T} \backslash\{b\}$ has at least three components); indeed, at least on an intuitive level, cutting $\mathbf{T}$ in a branch point should result in branches with reduced topological or metric complexity. This was exactly the procedure in the recent paper BT20, where topological characterizations of metric trees were given. We also use this idea in our forthcoming paper [BM20]. However, in the present context, cutting our given qc-tree $\mathbf{T}$ at a branch point $b$ leads to the problem that we cannot expect good uniform control for the size of the components of $\mathbf{T} \backslash\{b\}$, because some of these components might be very small.

For this reason, we cut our given qc-tree $\mathbf{T}$ at double points $v \in \mathbf{T}$, i.e., points $v$ such that $\mathbf{T} \backslash\{v\}$ has precisely two components. These double points $v$ are chosen so that the two components of $\mathbf{T} \backslash\{v\}$ are not too small and so that $v$ stays away from the branch points of $\mathbf{T}$ in 
a precise quantitative way (see $(4.1)$ and $(4.2)$; the relevant definitions can be found in (2.1) and (2.2)).

The paper is organized as follows. In Section 2 we review some basic topological facts about trees. We also show that in a tree $\mathbf{T}$ of bounded turning one can replace the original metric up to bi-Lipschitz equivalence by a diameter metric $d$. It is characterized by the property that $\operatorname{diam}[x, y]=d(x, y)$ for all $x, y \in \mathbf{T}$. The change to a diameter metric will allow us to make some simplifications of our arguments. In Section 3 we will prove a general fact of independent interest: if on an arc some points cast a "shadow" satisfying suitable conditions, then one can always find a "place in the sun". We use this to find double points in a qc-tree $\mathbf{T}$ with quantitative separation from branch points (see Proposition 3.1).

In Section 4 we introduce the somewhat technical concept of a $(\beta, \gamma)$ good double point at scale $\Delta>0$. We show that with suitable choices of the parameters cutting the qc-tree $\mathbf{T}$ in a maximal $\Delta$-separated set of $(\beta, \gamma)$-good double points at scale $\Delta>0$ results in pieces that have diameter comparable to $\Delta$ (Proposition 4.2 ). This fact is used in Section 5 to define the subdivisions of $\mathbf{T}$ into tiles as discussed above. We record various statements about the geometric properties of these tile decompositions. Weights of tiles are then defined in Section 6 . There we establish the facts about weights that are needed later on. In Section 7 we define the metric $\varrho$ and show that it is geodesic. The proof of Theorem 1.2 is then completed in Section 8 and the proof of Theorem 1.3 is given in Section 9. We conclude with remarks and open problems in Section 10 .

1.1. Notation. We summarize some notation used throughout this paper.

When an object $A$ is defined to be another object $B$, we write $A:=B$ for emphasis. Two non-negative quantities $a$ and $b$ are said to be comparable if there is a constant $C \geq 1$ (usually depending on some ambient parameters) such that

$$
\frac{1}{C} a \leq b \leq C a .
$$

We then write $a \asymp b$. The constant $C$ is referred to as $C(\asymp)$. Similarly, we write $a \lesssim b$ or $b \gtrsim a$, if there is a constant $C>0$ such that $a \leq C b$, and refer to the constant $C$ as $C(\lesssim)$ or $C(\gtrsim)$. If we want to emphasize the parameters $\alpha, \beta, \ldots$ on which $C$ depends, then we write $C=C(\alpha, \beta, \ldots)$.

We use the standard notation $\mathbb{N}=\{1,2, \ldots\}$ and $\mathbb{N}_{0}=\{0,1,2, \ldots\}$. 
The cardinality of a set $X$ is denoted by $\# X$ and the identity map on $X$ by $\operatorname{id}_{X}$. Let $(X, d)$ be a metric space, $a \in X$, and $r>0$. We denote by $B_{d}(a, r)=\{x \in X: d(a, x)<r\}$ the open ball and by $\bar{B}_{d}(a, r)=\{x \in X: d(a, x) \leq r\}$ the closed ball of radius $r$ centered at $a$. If $A, B \subset X$, we let $\operatorname{diam}_{d}(A)$ be the diameter, $\bar{A}$ be the closure of $A$ in $X$, int $(A)$ be the interior of $A$ in $X$, and

$$
\operatorname{dist}_{d}(A, B):=\inf \{d(x, y): x \in A, y \in B\}
$$

be the distance of $A$ and $B$. If $x \in X$, we set $\operatorname{dist}_{d}(x, A):=\operatorname{dist}_{d}(\{x\}, A)$. We drop the subscript $d$ from our notation for $B_{d}(a, r)$, etc., if the metric $d$ is clear from the context.

\section{Auxiliary faCts}

In this section we collect some auxiliary statements that will be used later.

Let $(X, d)$ be a metric space. A set $S \subset X$ is called $s$-separated for some $s>0$ if all distinct points $x, y \in S$ satisfy $d(x, y) \geq s$. Such a set $S$ is a maximal $s$-separated set if $S$ is not contained in a strictly larger subset of $X$ that is also $s$-separated. Every $s$-separated set $S \subset X$ is contained in a maximal s-separated set $S^{\prime} \subset X$. If $X$ is compact, then every $s$-separated set $S \subset X$ must be finite.

If the space $(X, d)$ is doubling (as defined in the introduction), then for each $0<\lambda<1$ there is a number $N^{\prime}=N^{\prime}(\lambda, N) \in \mathbb{N}$ only depending on $\lambda$ and the doubling constant $N$ of $X$ such that the following condition is true: if $s>0$ and $S \subset X$ is a $\lambda s$-separated set contained in a ball $B(x, s)$ with $x \in X$, then $S$ contains at most $N^{\prime}$ points. Conversely, if this condition is true for some $0<\lambda<1$ and $N^{\prime} \in \mathbb{N}$, then $X$ is doubling with a doubling constant $N=N\left(\lambda, N^{\prime}\right)$ only depending on $\lambda$ and $N^{\prime}$ (see [He01, Exercise 10.17]).

The doubling property is preserved under quasisymmetries, and in particular under bi-Lipschitz maps; in general though, the doubling constant will change (see [He01, Theorem 10.18]).

An $\operatorname{arc} J \subset X$ is a set homeomorphic to the unit interval $[0,1] \subset \mathbb{R}$. A (metric) $\operatorname{arc}(J, d)$ is a metric space homeomorphic to $[0,1]$. The points $a, b \in J$ corresponding to $0,1 \in[0,1]$ are called the endpoints of $J$. We denote by $\partial J:=\{a, b\}$ the set of endpoints of $J$, and by $\operatorname{int}(J):=J \backslash \partial J$ the set of interior points of $J$.

We require an elementary lemma.

Lemma 2.1. Let $(J, d)$ be an arc and $n \geq 2$ be an integer. Then we can decompose $J$ into $n$ non-overlapping subarcs of equal diameter $\Delta \geq \frac{1}{n} \operatorname{diam}(J)$. 
More explicitly, decomposing $J$ into $n$ non-overlapping subarcs means that we can find $\operatorname{arcs} I_{1}, \ldots, I_{n} \subset J$ with pairwise disjoint interiors such $J=I_{1} \cup \cdots \cup I_{n}$.

Proof. The existence of a decomposition of $J$ into $n$ non-overlapping subarcs of equal diameter is proved in [Me11, Lemma 2.2] (see also [Kul94, Lemma 2] for a related statement in greater generality). If we denote this diameter by $\Delta>0$, then we must have $\operatorname{diam}(J) \leq n \Delta$ as follows from the triangle inequality.

We now summarize some simple facts about trees. There is a rich literature on the underlying topological spaces, usually called dendrites. We refer to [Wh63, Chapter V], [Kur68, Section $§ 51$ VI], [Na92, Chapter $\mathrm{X}]$, and the references in these sources for more on the subject.

By definition, a metrizable topological space $X$ is called a dendrite if $X$ is a Peano continuum (i.e., it is compact, connected, and locally connected), and $X$ does not contain any Jordan curve (i.e., a homeomorphic image of the unit circle). A dendrite is called non-degenerate if it contains more than one point. The following statement reconciles our notion of a metric tree with the notion of a dendrite.

Proposition 2.2. Let $\mathbf{T}$ be a metric space. Then $\mathbf{T}$ is a tree if and only if $\mathbf{T}$ is a non-degenerate dendrite.

Proof. " $\Rightarrow$ " If $\mathbf{T}$ is a tree, then it is a Peano continuum and contains more than one point. Moreover, $\mathbf{T}$ cannot contain a Jordan curve $J$. Indeed, if $\mathbf{T}$ contains the Jordan curve $J$, then any two distinct points $x, y \in J$ can be connected by at least two distinct arcs in $\mathbf{T}$, namely the two subarcs of $J$ with endpoints $x$ and $y$. This is impossible, because $\mathbf{T}$ is a tree. It follows that $\mathbf{T}$ is a non-degenerate dendrite.

" $\Leftarrow "$ Conversely, suppose $\mathbf{T}$ is a non-degenerate dendrite. Since $\mathbf{T}$ is a Peano continuum, it is arc-connected, i.e., for any two distinct points $x, y \in \mathbf{T}$ there exists an arc $\alpha \subset \mathbf{T}$ with endpoints $x$ and $y$ (see [Na92, Theorem 8.23]). This arc is unique, because if there exists an $\operatorname{arc} \beta \subset \mathbf{T}$ with $\beta \neq \alpha$ and endpoints $x$ and $y$, then it is easy to see that $\alpha \cup \beta \subset \mathbf{T}$ contains a Jordan curve. This is impossible, because $\mathbf{T}$ is a dendrite. It follows that $\mathbf{T}$ is indeed a tree.

Let $\mathbf{T}$ be a tree. Then for all points $x, y \in \mathbf{T}$ with $x \neq y$, there exists a unique arc in $\mathbf{T}$ joining $x$ and $y$, i.e., it has the endpoints $x$ and $y$. We use the notation $[x, y]$ for this unique arc. It is convenient to allow $x=y$ here. Then $[x, y]$ denotes a degenerate arc consisting only of the point $x=y$. Sometimes we want to remove one or both endpoints from 
the arc $[x, y]$. Accordingly, we define

$$
(x, y]:=[x, y] \backslash\{x\}, \quad[x, y):=[x, y] \backslash\{y\}, \quad(x, y):=[x, y] \backslash\{x, y\} .
$$

If $\gamma$ is the image of any path in $\mathbf{T}$ joining $x$ and $y$, then necessarily $[x, y] \subset \gamma$.

A subset $X$ of a tree $(\mathbf{T}, d)$ is called a subtree of $\mathbf{T}$ if $X$ equipped with the restriction of the metric $d$ is also a tree. One can show that $X \subset \mathbf{T}$ is a subtree of $\mathbf{T}$ if and only if $X$ contains at least two points and is closed and connected. See [BT20, Lemma 3.3] for a simple direct argument; to justify this, one can also invoke Proposition 2.2 and the fact that a closed and connected subset of a dendrite is a dendrite (see [Na92, Corollary 10.6]). If $X$ is a subtree of $\mathbf{T}$, then $[x, y] \subset X$ for all $x, y \in X$.

Lemma 2.3. Let $(\mathbf{T}, d)$ be a tree and $V \subset \mathbf{T}$ be a finite set. Then the following statements are true:

(i) Two points $x, y \in \mathbf{T} \backslash V$ lie in the same component of $\mathbf{T} \backslash V$ if and only if $[x, y] \cap V=\emptyset$.

(ii) If $U$ is a component of $\mathbf{T} \backslash V$, then $U$ is an open set and $\bar{U}$ is a subtree of $\mathbf{T}$ with $\partial \bar{U} \subset \partial U \subset V$.

(iii) If $U$ and $W$ and are distinct components of $\mathbf{T} \backslash V$, then $\bar{U}$ and $\bar{W}$ have at most one point in common. Such a common point belongs to $V$, and is a boundary point of both $\bar{U}$ and $\bar{W}$.

Proof. (i) Since $V \subset \mathbf{T}$ is a finite set, it is closed in $\mathbf{T}$. So $\mathbf{T} \backslash V$ is an open subset of $\mathbf{T}$. Since $\mathbf{T}$ is locally connected, each component $U$ of $\mathbf{T} \backslash V$ is open. Moreover, as an open and connected subset of the Peano continuum $\mathbf{T}$, such a component $U$ is arc-connected (see [Na92, Theorem 8.26]). So if two points $x, y \in \mathbf{T} \backslash V$ lie in the same component $U$ of $\mathbf{T} \backslash V$, then there exists an arc $\gamma$ in $U$ joining $x$ and $y$. Then $\gamma=[x, y] \subset U$, and so $[x, y] \cap V=\emptyset$.

Conversely, if $x, y \in \mathbf{T} \backslash V$ and $[x, y] \cap V=\emptyset$, then $[x, y]$ is a connected subset of $\mathbf{T} \backslash V$. Hence there exists a component $U$ of $\mathbf{T} \backslash V$ with $[x, y] \subset U$; so $x$ and $y$ lie in the same component $U$ of $\mathbf{T} \backslash V$.

(ii) If $U$ is a component of $\mathbf{T} \backslash V$, then $U$ is an open set (as we have seen in the proof of (i) and $\bar{U}$ is a subtree of $\mathbf{T}$ (as follows from the characterization of subtrees discussed before the lemma).

The inclusion $\partial \bar{U} \subset \partial U$ is true for all sets $U \subset \mathbf{T}$. It remains to show $\partial U \subset V$. Indeed, if $x \in \partial U$, then $x$ cannot belong to $U$ (since $U$ is open) or any other component $W$ of $\mathbf{T} \backslash V$ (because otherwise $U \cap W \neq \emptyset)$; so $x$ lies in the complement of $\mathbf{T} \backslash V$ in $\mathbf{T}$, i.e., $x \in V$. 
(iii) Suppose $U$ and $W$ are distinct components of $\mathbf{T} \backslash V$. Since $U$ and $W$ are disjoint open subsets of $\mathbf{T}$ by (ii), no interior point of $\bar{U}$ can belong to $\bar{W}$, and no interior point of $\bar{W}$ can belong to $\bar{U}$. Hence

$$
\bar{U} \cap \bar{W}=\partial \bar{U} \cap \bar{W}=\partial \bar{U} \cap \partial \bar{W} \subset \partial U \cap \partial W \subset V
$$

by (ii). In particular, $\bar{U} \cap \bar{W}$ is a subset of the finite set $V$, and any point in $\bar{U} \cap \bar{W}$ must be a boundary point of both $\bar{U}$ and $\bar{W}$.

Actually, $\bar{U} \cap \bar{W}$ consists of at most one point; otherwise, $\bar{U} \cap \bar{W}$ contains two distinct points $x$ and $y$, and hence the infinite set $[x, y]$, because $\bar{U}$ and $\bar{W}$ are subtrees of $\mathbf{T}$. This is impossible, because the set $\bar{U} \cap \bar{W} \subset V$ is finite.

Let $\mathbf{T}$ be a tree, $p \in \mathbf{T}$, and $U$ be a component of $\mathbf{T} \backslash\{p\}$. Then $U \neq \mathbf{T}$ is open, and so $\partial U \neq \emptyset$, because $\mathbf{T}$ is connected. So by Lemma 2.3 (ii) we have $\emptyset \neq \partial U \subset\{p\}$. Hence $\partial U=\{p\}$ and so $\bar{U}=U \cup\{p\}$. Then $B:=\bar{U}=U \cup\{p\}$ is a subtree of $\mathbf{T}$, called a branch of $p$ (in $\mathbf{T})$.

The components $U$ of any open subset $W$ of a tree $\mathbf{T}$ form a null sequence in the following sense: for each $\epsilon>0$ there are only finitely many such components $U$ with $\operatorname{diam}(U) \geq \epsilon$. In particular, the number of components of $W$ is finite or countably infinite. This follows from a more general fact about open subsets of hereditarily locally connected metric continua; see Wh63, p. 90, Corollary (2.2)] or Kur68, p. 269, Theorem 3]. Note that we can apply this result by Proposition 2.2 and because every dendrite is hereditarily locally connected (this is explicitly stated in [Na92, Corollary 10.5] and follows from the fact, mentioned above, that every subcontinuum of a dendrite is a dendrite).

In particular, each point $p$ in a tree $\mathbf{T}$ can have at most countably many distinct complementary components $U$ and hence there are only countably many distinct branches $B$ of $p$. Only finitely many of these branches can have a diameter exceeding a given positive number (for a direct proof of these facts see also [BT20, Section 3]). This implies that we can label the branches $B_{n}$ of $p$ by numbers $n=1,2,3, \ldots$ so that

$$
\operatorname{diam}\left(B_{1}\right) \geq \operatorname{diam}\left(B_{2}\right) \geq \operatorname{diam}\left(B_{3}\right) \geq \ldots .
$$

If there are precisely two such branches, then we call $p$ a double point of $\mathbf{T}$ and define

$$
D_{\mathbf{T}}(p)=\operatorname{diam}\left(B_{2}\right) .
$$

So $D_{\mathbf{T}}(p)$ is the diameter of the smallest branch of a double point $p$. 
If there are at least three branches of $p$, then $p$ is called a branch point of $\mathbf{T}$. In this case, we set

$$
H_{\mathbf{T}}(p)=\operatorname{diam}\left(B_{3}\right) .
$$

So $H_{\mathbf{T}}(p)$ is the diameter of the third largest branch of $p$.

The following statement gives a criterion how to detect branch points.

Lemma 2.4. Let $(\mathbf{T}, d)$ be a tree, $b, x_{1}, x_{2}, x_{3} \in \mathbf{T}$ with $b \neq x_{1}, x_{2}, x_{3}$ and suppose that the sets $\left[x_{1}, b\right),\left[x_{2}, b\right),\left[x_{3}, b\right)$ are pairwise disjoint. Then the points $x_{1}, x_{2}, x_{3}$ lie in different components of $\mathbf{T} \backslash\{b\}$ and $b$ is a branch point of $\mathbf{T}$.

Proof. This is [BT20, Lemma 3.6]. For the reader's convenience we reproduce the argument. The arcs $\left[x_{1}, b\right]$ and $\left[x_{2}, b\right]=\left[b, x_{2}\right]$ have only the point $b$ in common. So their union $\left[x_{1}, b\right] \cup\left[b, x_{2}\right]$ is an arc and this arc must be equal to $\left[x_{1}, x_{2}\right]$. Hence $b \in\left[x_{1}, x_{2}\right]$ which by Lemma 2.3(i) implies that $x_{1}$ and $x_{2}$ lie in different components of $\mathbf{T} \backslash\{b\}$. A similar argument shows that $x_{3}$ must be contained in a component of $\mathbf{T} \backslash\{b\}$ different from the components containing $x_{1}$ and $x_{2}$. In particular, $\mathbf{T} \backslash\{b\}$ has at least three components and so $b$ is a branch point of $\mathbf{T}$. The statement follows.

The tree $(\mathbf{T}, d)$ is of $K$-bounded turning with $K \geq 1$ (as defined in the introduction) if and only if

$$
\operatorname{diam}[x, y] \leq K d(x, y)
$$

for all $x, y \in \mathbf{T}$. Here and in the following, diam $[x, y]$ instead of $\operatorname{diam}([x, y])$ denotes the diameter of the arc $[x, y]$; we omit the parentheses for better readability.

We define the diameter distance on $\mathbf{T}$ by

$$
\operatorname{dd}(x, y):=\operatorname{diam}[x, y]
$$

for $x, y \in \mathbf{T}$. We record some properties of this distance function.

Lemma 2.5. Let $(\mathbf{T}, d)$ be a metric tree. Then the following statements are true:

(i) dd is a metric on $\mathbf{T}$.

(ii) For each arc $J \subset \mathbf{T}$ we have

$$
\operatorname{diam}_{\mathrm{dd}}(J)=\operatorname{diam}(J),
$$

where diam $_{\mathrm{dd}}$ denotes the diameter with respect to $\mathrm{dd}$.

(iii) ( $\mathbf{T}, \mathrm{dd})$ is of 1-bounded turning.

(iv) $(\mathbf{T}, d)$ is of $K$-bounded turning for $K \geq 1$ if and only if the identity map $\mathrm{id}_{\mathbf{T}}:(\mathbf{T}, d) \rightarrow(\mathbf{T}, \mathrm{dd})$ is K-bi-Lipschitz. 
Proof. This is [Me11, Lemma 2.1], but we will include the simple proof for the convenience of the reader.

(i) All properties of a metric for dd are immediate except the triangle inequality which follows from the fact that if $x, y, z \in \mathbf{T}$, then $[x, z] \subset$ $[x, y] \cup[y, z]$.

(ii) For all $x, y \in J$, we have $d(x, y) \leq \mathrm{dd}(x, y)$, and so $\operatorname{diam}(J) \leq$ $\operatorname{diam}_{\mathrm{dd}}(J)$. Moreover, for all $x, y \in J$ we have $[x, y] \subset J$. Hence $\mathrm{dd}(x, y) \leq \operatorname{diam}(J)$; so $\operatorname{diam}_{\mathrm{dd}}(J) \leq \operatorname{diam}(J)$ and the statement follows.

(iii) This follows directly from (ii), since

$$
\mathrm{dd}(x, y)=\operatorname{diam}[x, y]=\operatorname{diam}_{\mathrm{dd}}[x, y]
$$

for all $x, y \in \mathbf{T}$.

(iv) If ( $\mathbf{T}, d)$ is of $K$-bounded turning, then for all $x, y \in \mathbf{T}$ we have

$$
\operatorname{dd}(x, y)=\operatorname{diam}[x, y] \leq K d(x, y) \leq K \mathrm{dd}(x, y) .
$$

Thus the identity map $\operatorname{id}_{\mathbf{T}}:(\mathbf{T}, d) \rightarrow(\mathbf{T}, \mathrm{dd})$ is $K$-bi-Lipschitz. Conversely, if this map is $K$-bi-Lipschitz, then for all $x, y \in \mathbf{T}$,

$$
\operatorname{diam}[x, y]=\operatorname{diam}_{\mathrm{dd}}[x, y]=\mathrm{dd}(x, y) \leq K d(x, y) .
$$

Therefore, $(\mathbf{T}, d)$ is of $K$-bounded turning.

We say a metric $d$ on a metric tree $\mathbf{T}$ is a diameter metric if $d(x, y)=$ $\operatorname{diam}[x, y]$ for all $x, y \in \mathbf{T}$. In this case, $d=\mathrm{dd}$, where $\mathrm{dd}$ is defined as in 2.3 .

Suppose $(\mathbf{T}, d)$ is a qc-tree, i.e., a tree that is doubling and of bounded turning. Then the previous lemma implies that $(\mathbf{T}, \mathbf{d d})$ is bi-Lipschitz equivalent, and in particular quasisymmetrically equivalent, to $(\mathbf{T}, d)$. Moreover, $(\mathbf{T}, \mathbf{d d})$ is of 1-bounded turning and also doubling, since the latter condition is invariant under bi-Lipschitz equivalence; so ( $\mathbf{T}, \mathbf{d d})$ is also a qc-tree. This implies that in order to prove Theorems 1.2 and 1.3 , we are reduced to the case that the qc-tree in question carries a diameter metric. This reduction makes the proofs somewhat easier, but we still face major problems, because there is no obvious way to turn a diameter metric into a geodesic metric by a quasisymmetry.

For the rest of the paper we will assume that $(\mathbf{T}, d)$ is a qc-tree that is equipped with a diameter metric $d$. Nothing essential changes if we rescale the metric. So we may also assume that $\operatorname{diam}(\mathbf{T})=1$. We will denote the doubling constant of $\mathbf{T}$ by $N$ throughout the paper. 


\section{SUN AND SHADOW}

In this section we will prove a statement, Proposition 3.1, that will allow us to find double points in our given qc-tree $\mathbf{T}$ that stay away from the branch points of $\mathbf{T}$ in a geometrically controlled manner. In the formulation of the proposition, we use the function defined in $(2.2)$.

Proposition 3.1. There exists a constant $\gamma=\gamma(N)>0$ only depending on the doubling constant $N$ of $\mathbf{T}$ with the following property: if $\Delta>0$ and $J \subset \mathbf{T}$ is an arc with $\operatorname{diam}(J) \geq \Delta$, then there exists a double point $x \in J$ of $\mathbf{T}$ such that

$$
d(x, b) \geq \gamma \min \left\{H_{\mathbf{T}}(b), \Delta\right\}
$$

for all branch points $b \in \mathbf{T}$.

To prove this statement, we require two auxiliary facts.

Lemma 3.2. Let $(J, d)$ be a metric arc equipped with a diameter metric $d, J^{\prime} \subset J$ be an arc, and $A \subset J$ be a set with $\#\left(A \cap J^{\prime}\right) \leq M$, where $M \in \mathbb{N}$. Then there exists an arc $I \subset J^{\prime}$ such that

$$
\operatorname{diam}(I)=\frac{1}{6 M} \operatorname{diam}\left(J^{\prime}\right) \text { and } \operatorname{dist}\left(I, A \cup \partial J^{\prime}\right) \geq \frac{1}{6 M} \operatorname{diam}\left(J^{\prime}\right) .
$$

The statement is somewhat technical, because three arcs $I \subset J^{\prime} \subset J$ are involved, but in this form the lemma will be useful for us later on.

Proof of Lemma 3.2. The construction that follows is illustrated in Figure 1. By Lemma 2.1] we can decompose $J^{\prime}$ into $M+1$ non-overlapping $\operatorname{arcs} J_{1}^{\prime}, \ldots, J_{M+1}^{\prime}$ of equal diameter $\Delta$.

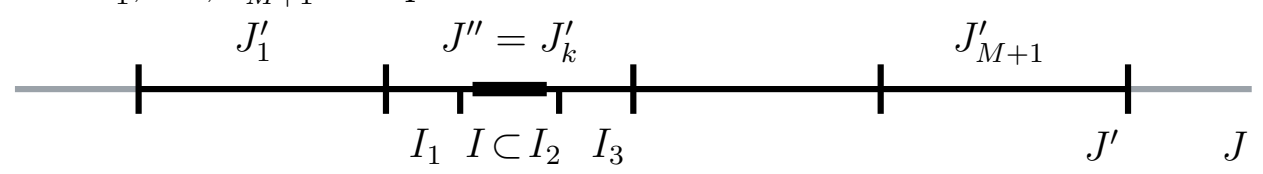

Figure 1. The arcs in the proof of Lemma 3.2 .

We have

$$
\Delta \geq \frac{1}{M+1} \operatorname{diam}\left(J^{\prime}\right) \geq \frac{1}{2 M} \operatorname{diam}\left(J^{\prime}\right) .
$$

Since $\#\left(A \cap J^{\prime}\right) \leq M$ and $J_{1}^{\prime}, \ldots, J_{M+1}^{\prime}$ have pairwise disjoint interiors, by the pigeon-hole principle there exists $k \in\{1, \ldots, M+1\}$ such that for $J^{\prime \prime}:=J_{k}^{\prime}$ we have $\operatorname{int}\left(J^{\prime \prime}\right) \cap A=\emptyset$. We subdivide $J^{\prime \prime}$ into three non-overlapping arcs $I_{1}, I_{2}, I_{3}$ of equal diameter. Then

$$
\operatorname{diam}\left(I_{i}\right) \geq \frac{1}{3} \operatorname{diam}\left(J^{\prime \prime}\right) \geq \frac{1}{6 M} \operatorname{diam}\left(J^{\prime}\right)
$$

for $i=1,2,3$. We may assume that $I_{1}$ contains one endpoint of $J^{\prime \prime}, I_{3}$ contains the other endpoint, and $I_{2}$ is the "middle" arc in the decomposition of $J^{\prime \prime}$. It easily follows from (3.1) and the intermediate value theorem that there exists an arc $I \subset I_{2}$ with $\operatorname{diam}(I)=\frac{1}{6 M} \operatorname{diam}\left(J^{\prime}\right)$. 
If $a \in A \cup \partial J^{\prime}$, then $a \notin \operatorname{int}\left(J^{\prime \prime}\right)$. So if we travel from a point $x \in I$ to the point $a$ along $[x, a] \subset J$, we must traverse $I_{1}$ or $I_{3}$. Since $d$ is a diameter metric, (3.1) implies that

$$
d(x, a)=\operatorname{diam}[x, a] \geq \min \left\{\operatorname{diam}\left(I_{1}\right), \operatorname{diam}\left(I_{3}\right)\right\} \geq \frac{1}{6 M} \operatorname{diam}\left(J^{\prime}\right) .
$$

Hence $\operatorname{dist}\left(I, A \cup \partial J^{\prime}\right) \geq \frac{1}{6 M} \operatorname{diam}\left(J^{\prime}\right)$. The statement follows.

Lemma 3.3 (Ein Platz an der Sonne ${ }^{1}$ ). Let $(J, d)$ be a metric arc equipped with a diameter metric $d$, and $S: J \rightarrow[0, \operatorname{diam}(J)]$ be a function. Suppose that there is a constant $M \in \mathbb{N}$ such that for all subarcs $I \subset J$ we have

$$
\#\{p \in I: S(p) \geq \operatorname{diam}(I)\} \leq M .
$$

Then there exists a constant $\sigma=\sigma(M)>0$ and a point $x \in J$ such that $d(x, p) \geq \sigma S(p)$ for all $p \in J$.

In other words, the set $J \backslash \bigcup_{p \in J} B(p, \sigma S(p))$ is non-empty (here we use the convention that $B(p, 0)=\emptyset)$. If we think of each point $p \in J$ with $S(p)>0$ as "casting a shadow" of radius $\sigma S(p)$ around $p$, then the lemma says that the union of all shadows does not cover $J$, and so there is a "place in the sun".

Proof. Without loss of generality we may assume that $\operatorname{diam}(J)=1$. Consider the set $A:=\{p \in J: S(p)>0\}$. Let $\lambda:=1 /(6 M)$ and define $A_{n}:=\left\{p \in A: S(p) \geq \lambda^{n}\right\}$ for $n \in \mathbb{N}_{0}$. Obviously, $A_{n} \subset A_{n+1}$ for $n \in \mathbb{N}_{0}$ and $A=\bigcup_{n \in \mathbb{N}_{0}} A_{n}$. We will inductively define arcs $J_{n} \subset J$ for $n \in \mathbb{N}_{0}$ such that $J_{0} \supset J_{1} \supset J_{2} \ldots, \operatorname{diam}\left(J_{n}\right)=\lambda^{n}$ for all $n \in \mathbb{N}_{0}$, and $\operatorname{dist}\left(J_{n}, A_{n-1}\right) \geq \lambda^{n}$ for all $n \in \mathbb{N}$.

We set $J_{0}:=J$. Suppose $\operatorname{arcs} J_{0}, \ldots, J_{n}$ with the desired properties have already been defined for some $n \in \mathbb{N}_{0}$. Then by our hypotheses $\#\left(A_{n} \cap J_{n}\right) \leq M$. It follows from Lemma 3.2 that we can find an arc $J_{n+1} \subset J_{n}$ with

$$
\operatorname{diam}\left(J_{n+1}\right)=\frac{1}{6 M} \operatorname{diam}\left(J_{n}\right)=\lambda \operatorname{diam}\left(J_{n}\right)=\lambda^{n+1}
$$

and $\operatorname{dist}\left(J_{n+1}, A_{n}\right) \geq \lambda^{n+1}$. Hence $J_{n+1}$ has the desired properties, and we can continue the process indefinitely.

We have $\bigcap_{n \in \mathbb{N}_{0}} J_{n} \neq \emptyset$, and so we can pick a point $x \in J$ that lies in all arcs $J_{n}$. If $p \in A$ is arbitrary, then there exists a smallest $n \in \mathbb{N}_{0}$ such that $p \in A_{n}$. Then $S(p) \in\left[\lambda^{n}, \lambda^{n-1}\right)$, and so

$$
d(x, p) \geq \operatorname{dist}\left(x, A_{n}\right) \geq \lambda^{n+1} \geq \lambda^{2} S(p) .
$$

So if we choose $\sigma=\lambda^{2}=1 /\left(36 M^{2}\right)$, then $x$ is a point as desired.

\footnotetext{
${ }^{1}$ Mit fünf Mark sind Sie dabei!
} 
Proof of Proposition 3.1. Let $\Delta>0$ and suppose $J \subset \mathbf{T}$ is an arc with $\operatorname{diam}(J) \geq \Delta$. Then $J=[u, v]$, where $u, v \in \mathbf{T}$ are the endpoints of $J$. We set $S(p)=\Delta$ for $p \in\{u, v\}, S(p)=\min \left\{H_{\mathbf{T}}(p), \Delta\right\}$ for a branch point $p \in(u, v)$, and $S(p)=0$ for all other points $p \in(u, v)$. Since $\operatorname{diam}(J) \geq \Delta$ and $0 \leq S(p) \leq \Delta$ for $p \in J$, we can consider $S$ as a function $S: J \rightarrow[0, \operatorname{diam}(J)]$.

Claim. There exists a constant $M=M(N) \in \mathbb{N}$ such that for all $\operatorname{arcs} I \subset J$ we have

$$
\#\{p \in I: S(p) \geq \operatorname{diam}(I)\} \leq M .
$$

In other words, $S$ satisfies the hypotheses of Lemma 3.3 with a constant $M=M(N)$ only depending on the doubling constant $N$ of $\mathbf{T}$.

To see this, fix an arc $I \subset J$ and let $R:=\{p \in \operatorname{int}(I): S(p) \geq \rho\}$, where $\rho:=\operatorname{diam}(I)>0$. Each point $r \in R$ is a branch point of $\mathbf{T}$ and there exists a large component $U_{r}$ of $\mathbf{T} \backslash\{r\}$ that is disjoint from $I$, but attached to $I$ through the point $r$. There are \#R such components. The doubling property then gives a bound on $\# R$ only depending on $N$. In the following we present the details of this argument, illustrated in Figure 2 .

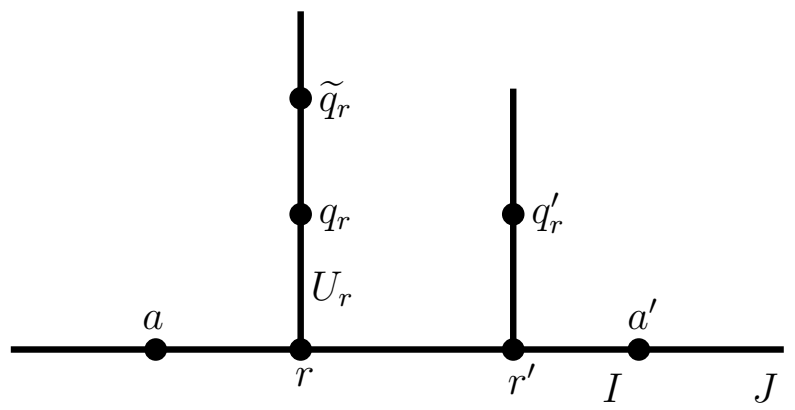

FiguRE 2. Bounding the number of elements in $R$.

We have $I=\left[a, a^{\prime}\right]$, where $a, a^{\prime} \in I$ are the endpoints of $I$. Consider an arbitrary point $r \in R \subset \operatorname{int}(I)=\left(a, a^{\prime}\right)$. Then $r$ is a branch point of $\mathbf{T}$ with $H_{\mathbf{T}}(r) \geq S(r) \geq \rho>0$. Each of the connected sets $[a, r)$ and $\left(r, a^{\prime}\right]$ is contained in a component of $\mathbf{T} \backslash\{r\}$. Hence there must be another component $U_{r}$ of $\mathbf{T} \backslash\{r\}$ with $\operatorname{diam}\left(U_{r}\right) \geq H_{\mathbf{T}}(r) \geq \rho$ that is disjoint from $I=[a, r) \cup\{r\} \cup\left(r, a^{\prime}\right]$. There exists a point $\widetilde{q}_{r} \in U_{r}$ with $d\left(\widetilde{q}_{r}, r\right) \geq \operatorname{diam}\left(U_{r}\right) / 2 \geq \rho / 2$; otherwise, $\bar{U}_{r}=U_{r} \cup\{r\} \subset B(r, \rho / 2)$ and so $\operatorname{diam}\left(U_{r}\right) \leq \operatorname{diam}\left(\bar{U}_{r}\right)<\rho$, which is a contradiction.

Then $\left(r, \widetilde{q}_{r}\right] \subset U_{r}$, and it easily follows from the intermediate value theorem that we can find a point $q_{r} \in\left(r, \widetilde{q}_{r}\right] \subset U_{r}$ with $d\left(q_{r}, r\right)=\rho / 2$. We have

$$
d\left(a, q_{r}\right) \leq d(a, r)+d\left(r, q_{r}\right) \leq \operatorname{diam}(I)+\rho / 2=3 \rho / 2,
$$


and so $q_{r} \in \bar{B}(a, 3 \rho / 2)$.

If $r, r^{\prime} \in R$ with $r \neq r^{\prime}$, then the corresponding points $q_{r}$ and $q_{r^{\prime}}$ lie in different components of $\mathbf{T} \backslash\left\{r^{\prime}\right\}$. To see this, note that $\bar{U}_{r}=U_{r} \cup\{r\}$ is a connected set with

$$
\bar{U}_{r}=U_{r} \cup\{r\} \subset(\mathbf{T} \backslash I) \cup\left(\mathbf{T} \backslash\left\{r^{\prime}\right\}\right) \subset \mathbf{T} \backslash\left\{r^{\prime}\right\},
$$

and so $\bar{U}_{r}$ is contained in a component of $\mathbf{T} \backslash\left\{r^{\prime}\right\}$. In particular, $q_{r} \in U_{r}$ and $r \in I \backslash\left\{r^{\prime}\right\}$ lie in the same component of $\mathbf{T} \backslash\left\{r^{\prime}\right\}$. On the other hand, $q_{r^{\prime}}$ was chosen from a component $U_{r^{\prime}}$ of $\mathbf{T} \backslash\left\{r^{\prime}\right\}$ that does not contain any point of $I$.

Since $q_{r}$ and $q_{r^{\prime}}$ lie in different components of $\mathbf{T} \backslash\left\{r^{\prime}\right\}$, Lemma 2.3 (i) implies that $r^{\prime} \in\left[q_{r}, q_{r^{\prime}}\right]$. In particular,

$$
d\left(q_{r}, q_{r^{\prime}}\right)=\operatorname{diam}\left[q_{r}, q_{r^{\prime}}\right] \geq d\left(r^{\prime}, q_{r^{\prime}}\right)=\rho / 2 .
$$

So the points $q_{r}, r \in R$, have pairwise mutual distance $\geq \rho / 2$ and are all contained in the ball $\bar{B}(a, 3 \rho / 2)$. It follows that $\# R$ is bounded by a constant only depending on $N$ (see the discussion in the beginning of Section 2). Since the endpoints of $I$ are not contained in $R$, we have to possibly increase this bound by 2 to obtain a bound as in $(3.3)$ with a constant $M=M(N)$. The Claim follows.

Lemma 3.3 now guarantees the existence of a point $x \in J$ such that

$$
d(x, p) \geq \sigma S(p)
$$

for all $p \in J$, where $\sigma=\sigma(M)=\sigma(N)>0$ can be chosen to depend only on $M$ and hence on $N$. We may assume that $0<\sigma \leq 1$, and so $\sigma^{2} \leq \sigma$.

We claim that the statement of the proposition is true with $\gamma:=\sigma^{2} / 2$ which only depends on $N$. To see this, let $b \in \mathbf{T}$ be an arbitrary branch point of $\mathbf{T}$. As we travel from $b$ to $x$ along the arc $[b, x]$, there is a first point $r \in J$. We now consider two cases depending on the location of $r$.

Case 1. $r \in B(u, \sigma \Delta / 2) \cup B(v, \sigma \Delta / 2)$. In this case, we may assume $r \in B(u, \sigma \Delta / 2)$. Since $S(u)=\Delta$, by choice of $x$ we then have

$$
\begin{aligned}
d(x, b) & =\operatorname{diam}[x, b] \geq d(x, r) \geq d(x, u)-d(r, u) \\
& \geq \sigma S(u)-\sigma \Delta / 2=\sigma \Delta / 2 \geq \sigma \min \left\{H_{\mathbf{T}}(b), \Delta\right\} / 2 \\
& \geq \gamma \min \left\{H_{\mathbf{T}}(b), \Delta\right\} .
\end{aligned}
$$

This is the desired inequality in this case.

Case 2. $r \notin B(u, \sigma \Delta / 2) \cup B(v, \sigma \Delta / 2)$. Then in particular $r \in \operatorname{int}(J)$. There exists a component $U$ of $\mathbf{T} \backslash\{b\}$ that is disjoint from $J$ and 


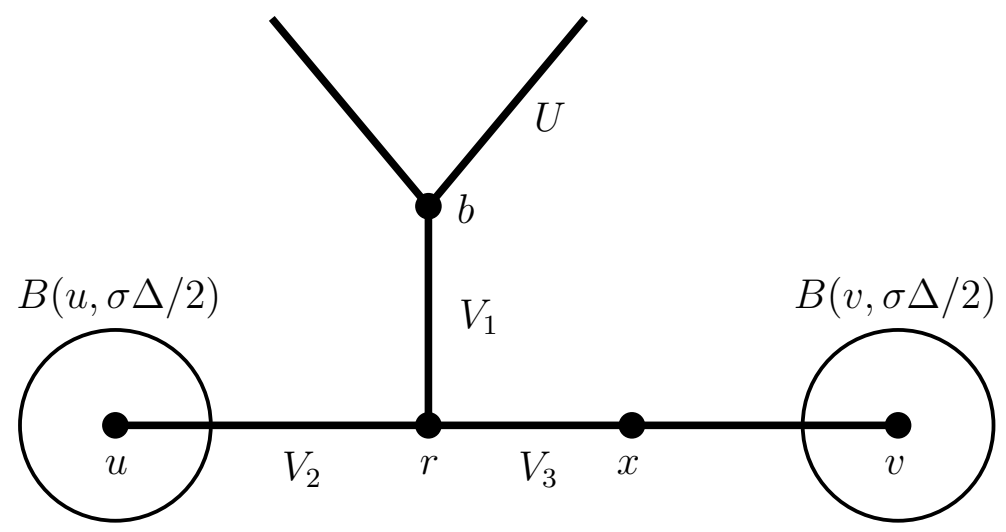

Figure 3. The estimate of Case 2.

satisfies $\operatorname{diam}(U) \geq H_{\mathbf{T}}(b)$. This connected set does not contain $r \in J$ and so it is contained in a component $V_{1}$ of $\mathbf{T} \backslash\{r\}$. Hence

$$
\operatorname{diam}\left(V_{1}\right) \geq \operatorname{diam}(U) \geq H_{\mathbf{T}}(b) .
$$

Two other components $V_{2}$ and $V_{3}$ of $\mathbf{T} \backslash\{r\}$ contain the half-open (and non-empty) arcs $[u, r)$ and $[v, r)$, respectively. The situation is illustrated in Figure 3. It follows that

$$
\operatorname{diam}\left(V_{2}\right) \geq \operatorname{diam}[u, r) \geq d(u, r) \geq \sigma \Delta / 2 .
$$

Here we have used that $r \notin B(u, \sigma \Delta / 2)$. Similarly, $\operatorname{diam}\left(V_{3}\right) \geq \sigma \Delta / 2$, and so

$$
\begin{aligned}
H_{\mathbf{T}}(r) & \geq \min \left\{\operatorname{diam}\left(V_{i}\right): i \in\{1,2,3\}\right\} \\
& \geq \min \left\{H_{\mathbf{T}}(b), \sigma \Delta / 2\right\} \geq \frac{\sigma}{2} \min \left\{H_{\mathbf{T}}(b), \Delta\right\} .
\end{aligned}
$$

It follows that

$$
\begin{aligned}
d(x, b) & =\operatorname{diam}[x, b] \geq d(x, r) \geq \sigma S(r)=\sigma \min \left\{H_{\mathbf{T}}(r), \Delta\right\} \\
& \geq \sigma^{2} \min \left\{H_{\mathbf{T}}(b), \Delta\right\} / 2=\gamma \min \left\{H_{\mathbf{T}}(b), \Delta\right\},
\end{aligned}
$$

as desired.

Note that $x \in J$ is a double point of $\mathbf{T}$. Indeed, $x$ is not a branch point of $\mathbf{T}$, because $x$ has a positive distance to each of them. On the other hand, $d(x, u) \geq \sigma S(u)=\sigma \Delta>0$, and so $x \neq u$. Similarly, $x \neq v$. Since $x \in[u, v]=J$, the points $u$ and $v$ lie in different components of $\mathbf{T} \backslash\{x\}$ by Lemma 2.3 (i). In particular, there are at least two, but not more than two such components. Hence $x$ is a double point of $\mathbf{T}$.

With some small changes in the previous proof one can show that the set of double points $x \in J$ that satisfy the estimate in Proposition 3.1 is not only non-empty, but in a suitable sense actually fairly large 
(namely, uniformly perfect). Such a statement was proved in recent work by Lin and Rohde (see [LR18, Lemma 4.5]).

\section{Good Double points}

In this section we introduce the concept of a "good" double point of our given qc-tree $\mathbf{T}$. Attached to this concept are certain numerical parameters. The goal of this section is to show that with appropriate choices of these parameters, one can use a maximal set $V$ of good double points to obtain a decomposition of $\mathbf{T}$ with some desired geometric properties (see Proposition 4.2).

We fix a scale $0<\Delta \leq \operatorname{diam}(\mathbf{T})=1$. We consider double points $x \in \mathbf{T}$ with the property that both components of $\mathbf{T} \backslash\{x\}$ are large, meaning that

$$
D_{\mathbf{T}}(x) \geq \beta \Delta
$$

for some constant $\beta \geq 1\left(D_{\mathbf{T}}\right.$ was defined in (2.1) $)$. We will choose $\beta$ according to the following statement.

Proposition 4.1. There is a constant $\beta=\beta(N) \geq 1$ only depending on the doubling constant $N$ of $\mathbf{T}$ such that the following statement is true: if $V \subset \mathbf{T}$ is a set of double points of $\mathbf{T}$ that are $\Delta$-separated and satisfy (4.1), then either

(i) for each component $X$ of $\mathbf{T} \backslash V$ we have

$$
\operatorname{diam}(X) \leq 3 \beta \Delta
$$

or

(ii) there is an arc $I \subset \mathbf{T}$ with

$$
\operatorname{diam}(I) \geq \Delta \text { and } \operatorname{dist}(I, V) \geq \Delta,
$$

and such that (4.1) holds for each double point $x \in I$ of $\mathbf{T}$.

Proposition 3.1 implies that each arc $I \subset \mathbf{T}$ contains double points of $\mathbf{T}$. So in case (ii) of Proposition 4.1, we can add a double point $x \in I$ of $\mathbf{T}$ to $V$. Then this new set $V^{\prime}=V \cup\{x\}$ is again a set of double points of $\mathbf{T}$ that are $\Delta$-separated and satisfy (4.1). This implies that for a maximal set $V$ as in the proposition, statement (i) will always be true.

Proof of Proposition 4.1. By the doubling property, there exists a constant $N^{\prime}=N^{\prime}(N) \in \mathbb{N}$ only depending on the doubling constant $N$ of $\mathbf{T}$ with the following property: if $\rho>0$ and $B \subset \mathbf{T}$ is a ball in $\mathbf{T}$ of radius $6 \rho$, then every $\rho$-separated subset of $B$ contains at most $N^{\prime}$ points. We will show that the proposition is true with the constant $\beta=6 N^{\prime}$, which only depends on $N$. 


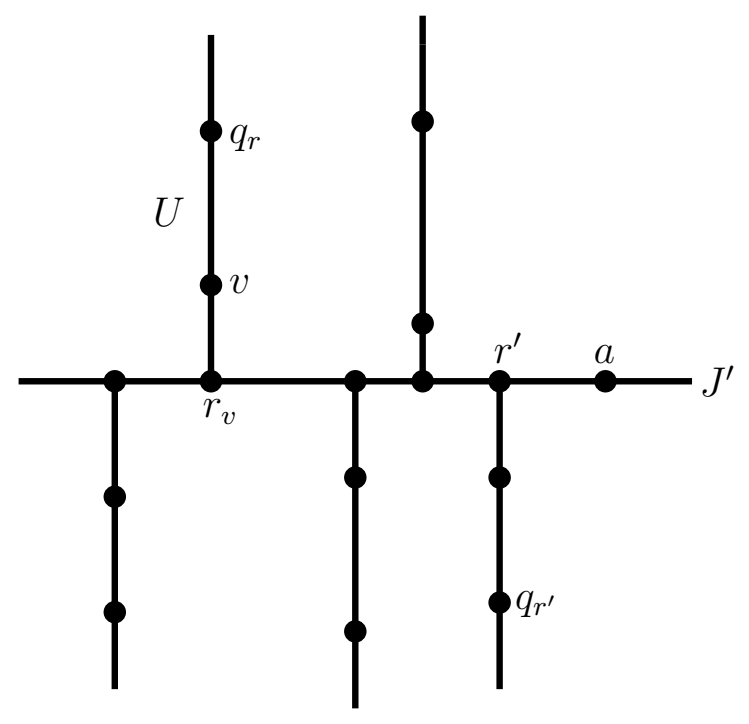

Figure 4. Roots in $J^{\prime}$.

Let $V \subset \mathbf{T}$ be a set as in the statement. Note that $V$ is a finite set, because $V$ is $\Delta$-separated and $\mathbf{T}$ is compact. If all components $X$ of $\mathbf{T} \backslash V$ satisfy (i), we are done. Otherwise, there exists a component $X$ of $\mathbf{T} \backslash V$ with $\operatorname{diam}(X)>3 \beta \Delta$. Then we can find points $z, w \in X$ with $d(z, w) \geq 3 \beta \Delta$. By Lemma 2.3 (i) we then have $[z, w] \cap V=\emptyset$ which implies that $J:=[z, w] \subset X$.

Note that $\operatorname{diam}(J) \geq d(z, w) \geq 3 \beta \Delta$. By decomposing $J$ into three non-overlapping subarcs of equal diameter $\geq \beta \Delta$ and trimming the "middle" arc to appropriate size, we can find an arc $J^{\prime} \subset J \subset X$ with $\operatorname{diam}\left(J^{\prime}\right)=\beta \Delta$ that has distance $\geq \beta \Delta$ from each of the two endpoints of $J$ (see the proof of Lemma 3.2 for a very similar argument). This implies that for every double point $x \in J^{\prime}$ of $\mathbf{T}$ the estimate (4.1) holds.

We want to find a subarc $I \subset J^{\prime} \subset \mathbf{T} \backslash V$ with $\operatorname{diam}(I) \geq \Delta$ and $\operatorname{dist}(I, V) \geq \Delta$. To this end, we fix a point $a \in J^{\prime}$ as "base point". Now suppose $v \in V$ is a point with $\operatorname{dist}\left(v, J^{\prime}\right)<\Delta$. If we travel from $v$ towards $a$ along $[v, a]$, there is a first point $r=r_{v}$ that belongs to $J^{\prime}$ (see Figure 4 for an illustration). Let

$$
R=\left\{r_{v}: v \in V, \operatorname{dist}\left(v, J^{\prime}\right)<\Delta\right\}
$$

be the set of these "root" points.

Claim. $\# R \leq N^{\prime}$.

To see this, first note that for each point $r \in R$ we can choose a point $v \in V$ with $d(v, r)<\Delta$ and $r=r_{v}$. The connected set $J^{\prime} \subset \mathbf{T} \backslash V \subset$ $\mathbf{T} \backslash\{v\}$ lies in one component of $\mathbf{T} \backslash\{v\}$. Then for the other component 
$U$ of $\mathbf{T} \backslash\{v\}$ we have $U \cap J^{\prime}=\emptyset$ and $\operatorname{diam}(U) \geq D_{\mathbf{T}}(v) \geq \beta \Delta$, because $v \in V$. Therefore, we can find a point $q \in U$ with $d(q, v)=\beta \Delta / 2$ (see the proof of Proposition 3.1 for more details in a similar claim). Define $v_{r}:=v$ and $q_{r}:=q$. We then have

$$
\begin{aligned}
d\left(q_{r}, a\right) & \leq d\left(q_{r}, v_{r}\right)+\operatorname{dist}\left(v_{r}, J^{\prime}\right)+\operatorname{diam}\left(J^{\prime}\right) \\
& <\beta \Delta / 2+\Delta+\beta \Delta \leq 3 \beta \Delta .
\end{aligned}
$$

Thus $q_{r} \in B(a, 3 \beta \Delta)$.

Moreover, if $r, r^{\prime} \in R$ are distinct, then the corresponding points $q_{r}$ and $q_{r^{\prime}}$ lie in different components of $\mathbf{T} \backslash\{r\}$. This can be justified by an argument similar to the one in the proof of Proposition 3.1. Hence $r \in\left[q_{r}, q_{r^{\prime}}\right]$ and so $\left[q_{r}, r\right] \subset\left[q_{r}, q_{r^{\prime}}\right]$. On the other hand, $q_{r}$ and $r$ lie in different components of $\mathbf{T} \backslash\left\{v_{r}\right\}$, and so $v_{r} \in\left[q_{r}, r\right] \subset\left[q_{r}, q_{r^{\prime}}\right]$. It follows that

$$
d\left(q_{r}, q_{r^{\prime}}\right)=\operatorname{diam}\left[q_{r}, q_{r^{\prime}}\right] \geq d\left(q_{r}, v_{r}\right) \geq \beta \Delta / 2 .
$$

This shows that the set $Q:=\left\{q_{r}: r \in R\right\}$ consists of $(\beta \Delta / 2)$-separated points and is contained in the ball $B(a, 3 \beta \Delta)$. The Claim now follows from the definition of the constant $N^{\prime}$.

By the Claim and Lemma 3.2 we can find an arc $I \subset J^{\prime}$ with

$$
\operatorname{diam}(I)=\frac{1}{6 N^{\prime}} \operatorname{diam}\left(J^{\prime}\right)=\frac{\beta}{6 N^{\prime}} \Delta=\Delta
$$

(by choice of $\left.\beta=6 N^{\prime}\right)$ and $\operatorname{dist}\left(I, R \cup \partial J^{\prime}\right) \geq \Delta$.

Then $\operatorname{dist}(I, V) \geq \Delta$. Indeed, let $v \in V$ be arbitrary. If $\operatorname{dist}\left(v, J^{\prime}\right) \geq$ $\Delta$, then clearly $\operatorname{dist}(v, I) \geq \operatorname{dist}\left(v, J^{\prime}\right) \geq \Delta$. If $\operatorname{dist}\left(v, J^{\prime}\right)<\Delta$, then as we travel from $v$ to a point in $I$ along an arc, we pass through $\partial J^{\prime}$ or the root point $r_{v} \in J^{\prime} \cap R$. So $\operatorname{dist}(v, I) \geq \operatorname{dist}\left(I, R \cup \partial J^{\prime}\right) \geq \Delta$ in this case as well.

Recall that $J^{\prime} \subset J$ was chosen such that every double point of $\mathbf{T}$ contained in $J^{\prime}$, and hence every such point contained in $I \subset J^{\prime}$, satisfies (4.1). Therefore, the arc $I$ has the desired properties and the statement follows.

In addition to (4.1), we want to choose double points $x$ of $\mathbf{T}$ that are separated from the branch points of $\mathbf{T}$ in a controlled way. More precisely, we require that

$$
d(x, b) \geq \gamma \min \left\{H_{\mathbf{T}}(b), \Delta\right\}
$$

for all branch points $b \in \mathbf{T}$. Here $\gamma=\gamma(N)$ is the constant from Proposition 3.1 that can be chosen to depend only on the doubling constant $N$ of $\mathbf{T}$. A double point $x \in \mathbf{T}$ is called $(\beta, \gamma)$-good at scale $\Delta$, if it satisfies 4.1 and 4.2 . 
Proposition 4.2. Let $\beta=\beta(N) \geq 1$ be the constant from Proposition 4.1. $\gamma=\gamma(N)>0$ be the constant from Proposition 3.1, and $0<\Delta \leq 1$

If $V \subset \mathbf{T}$ is a maximal $\Delta$-separated set of $(\beta, \gamma)$-good double points at scale $\Delta$, then

$$
\operatorname{diam}(X) \leq 3 \beta \Delta
$$

for each component $X$ of $\mathbf{T} \backslash V$.

Note that such a maximal set $V$ always exists, but we could very well have $V=\emptyset$. In this case, the statement says that $1=\operatorname{diam}(\mathbf{T}) \leq 3 \beta \Delta$. In other words, $V$ is necessarily non-empty if $0<\Delta<1 /(3 \beta)$.

Proof. Let $V$ be a set as in the statement. We argue by contradiction and assume that there is a component $X$ of $\mathbf{T} \backslash V$ with $\operatorname{diam}(X)>3 \beta \Delta$. Then we can find an arc $I \subset \mathbf{T}$ as in Proposition 4.1 (ii),

By Proposition 3.1 we can find a double point $x \in I$ of $\mathbf{T}$ such that

$$
d(x, b) \geq \gamma \min \left\{H_{\mathbf{T}}(b), \Delta\right\}
$$

for all branch points $b \in \mathbf{T}$. Then $x$ satisfies (4.1) and 4.2). Therefore, $x$ is a $(\beta, \gamma)$-good double point of $\mathbf{T}$ at scale $\Delta$. We also have

$$
\operatorname{dist}(x, V) \geq \operatorname{dist}(I, V) \geq \Delta \text {. }
$$

Hence $V^{\prime}=V \cup\{x\}$ is a $\Delta$-separated set consisting of $(\beta, \gamma)$-good double points at scale $\Delta$. Since $x \notin V$, this contradicts the maximality of $V$, and the statement follows.

At this point the importance of (4.2) is not at all obvious. The relevance of this condition will become apparent only later (see the remarks before Lemma 5.5.

\section{Subdividing THE TREE}

We want to subdivide our given qc-tree $\mathbf{T}$. As before, we may assume that $\mathbf{T}$ is equipped with a diameter metric $d$ and that $\operatorname{diam}(\mathbf{T})=1$. We fix constants $\beta \geq 1$ and $\gamma>0$ depending only on the doubling constant $N$ of $\mathbf{T}$ as in Proposition 4.2 , and a (small) constant $0<\delta<1 /(3 \beta)$.

Vertices and tiles. We will now inductively construct sets $\mathbf{V}^{n} \subset \mathbf{T}$ for $n \in \mathbb{N}$ such that

$$
\mathbf{V}^{1} \subset \mathbf{V}^{2} \subset \mathbf{V}^{3} \subset \ldots,
$$

where each $\mathbf{V}^{n}$ is a maximal $\delta^{n}$-separated set consisting of $(\beta, \gamma)$-good double points at scale $\delta^{n}$. Since $\mathbf{T}$ is compact, each set $\mathbf{V}^{n}$ will necessarily be finite. 
For $\mathbf{V}^{1}$ we choose a maximal $\delta$-separated subset of $\mathbf{T}$ consisting of $(\beta, \gamma)$-good double points at scale $\delta$. Suppose for some $n \in \mathbb{N}$, the sets $\mathbf{V}^{1} \subset \mathbf{V}^{2} \subset \ldots \subset \mathbf{V}^{n} \subset \mathbf{T}$ with the desired properties have been chosen. Then for $\Delta=\delta^{n+1} \leq \delta^{n}$ the set $\mathbf{V}^{n}$ is a $\Delta$-separated subset of $\mathbf{T}$ consisting of $(\beta, \gamma)$-good double points at scale $\Delta$. Hence it is contained in a maximal such set. We pick such a maximal set and denote it by $\mathbf{V}^{n+1}$. Clearly, $\mathbf{V}^{n} \subset \mathbf{V}^{n+1}$. It follows that we obtain sets $\mathbf{V}^{n}$ for all $n \in \mathbb{N}$ as desired. Since $\delta^{n} \leq \delta<1 /(3 \beta)$, we have $\mathbf{V}^{n} \neq \emptyset$ for each $n \in \mathbb{N}$, as follows from the remark after Proposition 4.2 .

Each point $v \in \mathbf{V}^{n}$ is called an $n$-vertex. The closure of a component of $\mathbf{T} \backslash \mathbf{V}^{n}$ is called an $n$-tile, and the set of all $n$-tiles is denoted by $\mathbf{X}^{n}$. We also speak of vertices and tiles if their level $n$ is clear from the context or irrelevant.

We now summarize some topological properties of vertices and tiles. Most of them are intuitively clear, often relying on the fact that each vertex is a double point, but we will include full proofs for the sake of completeness.

Lemma 5.1. For each $n \in \mathbb{N}$ the following statements are true:

(i) Each $n$-tile $X$ is a subtree of $\mathbf{T}$ with $\partial X \subset \mathbf{V}^{n}$.

(ii) If $X$ is an n-tile and $v \in \mathbf{V}^{n}$, then $X$ is contained in the closure of one of the two components of $\mathbf{T} \backslash\{v\}$ and disjoint from the other component.

(iii) If $X$ is an $n$-tile, then $\partial X \neq \emptyset$.

(iv) Two distinct $n$-tiles $X$ and $Y$ have at most one point in common. Such a common point is an n-vertex and a boundary point of both $X$ and $Y$.

(v) Each $n$-vertex $v$ is contained in precisely two distinct $n$-tiles $X$ and $Y$.

(vi) There are only finitely many $n$-tiles.

(vii) Each $(n+1)$-tile $X^{\prime}$ is contained in a unique $n$-tile $X$.

(viii) Each $n$-tile $X$ is equal to the union of all $(n+1)$-tiles $X^{\prime}$ with $X^{\prime} \subset X$.

(ix) If $v$ is n-vertex and $X$ an n-tile with $v \in X$, then $v \in \partial X$. Moreover, there exists precisely one $(n+1)$-tile $X^{\prime} \subset X$ with $v \in X^{\prime}$.

(x) If $X$ is an n-tile and $\partial X=\{v\} \subset \mathbf{V}^{n}$ is a singleton set, then $X=\bar{W}$, where $W$ is a component of $\mathbf{T} \backslash\{v\}$. 
Proof. (i) If $X$ is an $n$-tile, then $X=\bar{U}$, where $U$ is a component of $\mathbf{T} \backslash \mathbf{V}^{n}$. It then follows from Lemma 2.3 (ii) that $X=\bar{U}$ is a subtree of $\mathbf{T}$ with $\partial X=\partial \bar{U} \subset \partial U \subset \mathbf{V}^{n}$.

(ii) Again we have $X=\bar{U}$, where $U$ is a component of $\mathbf{T} \backslash \mathbf{V}^{n}$. Moreover, $v \in \mathbf{V}^{n}$ is a double point of $\mathbf{T}$, and so there exist precisely two components $W_{1}$ and $W_{2}$ of $\mathbf{T} \backslash\{v\}$. Since $U$ is a connected subset of $\mathbf{T} \backslash \mathbf{V}^{n} \subset \mathbf{T} \backslash\{v\}$, it is contained in one of these components, say $U \subset W_{1}$. Then $X=\bar{U} \subset \bar{W}_{1}=W_{1} \cup\{v\}$, and $X$ is disjoint from $W_{2}=\mathbf{T} \backslash \bar{W}_{1}$.

(iii) We have $\mathbf{V}^{n} \neq \emptyset$ and so it follows from (ii) that $X \neq \mathbf{T}$. Since $\mathbf{T}$ is connected, this implies that $\partial X \neq \emptyset$; otherwise, the non-empty set $X \neq \mathbf{T}$ would be an open and closed subset of the connected space $\mathbf{T}$. This is impossible.

(iv) This immediately follows from Lemma 2.3 (iii).

$(\mathrm{v})$ The point $v \in \mathbf{V}^{n}$ is a double point of $\mathbf{T}$. Hence there exist precisely two components $W_{1}$ and $W_{2}$ of $\mathbf{T} \backslash\{v\}$. We have $\bar{W}_{1}=$ $W_{1} \cup\{v\}$ and $\bar{W}_{2}=W_{2} \cup\{v\}$. Hence $v \in \bar{W}_{1} \cap \bar{W}_{2}$.

Lemma 2.3 (i) implies that for all points $x \in W_{1}$ and $y \in W_{2}$, we have $v \in[x, y]$. Since $v \in \bar{W}_{1} \cap \bar{W}_{2}$, we can choose $x$ and $y$ so close to $v$ that $[x, y]$ contains no other point in $\mathbf{V}^{n}$. Then $[x, v)$ is a connected subset of $\mathbf{T} \backslash \mathbf{V}^{n}$, and so it must be contained in a component $U_{1}$ of $\mathbf{T} \backslash \mathbf{V}^{n}$. Hence $X:=\bar{U}_{1}$ is an $n$-tile that contains the arc $[x, v]$, and so $v \in X$. Similarly, $(v, y]$ is contained in a component $U_{2}$ of $\mathbf{T} \backslash \mathbf{V}^{n}$, and $v$ is contained in the $n$-tile $Y:=\bar{U}_{2}$.

Since $v \in[x, y]$, by Lemma 2.3 (i) the components $U_{1}$ and $U_{2}$ of $\mathbf{T} \backslash \mathbf{V}^{n}$ containing $x$ and $y$, respectively, must be distinct. So $U_{1} \neq U_{2}$, and these sets are disjoint. Since $U_{1}$ and $U_{2}$ are open by Lemma 2.3(ii), the sets $X=\bar{U}_{1}$ and $U_{2}$ are also disjoint. Since $\emptyset \neq U_{2} \subset \bar{U}_{2}=Y$, we conclude that $X=\bar{U}_{1} \neq \bar{U}_{2}=Y$. So $v$ is contained in at least two distinct $n$-tiles $X$ and $Y$.

Suppose $Z=\bar{U}$ is another $n$-tile with $v \in Z$, where $U$ is a component of $\mathbf{T} \backslash \mathbf{V}^{n}$. A point $z \in U$ must be contained in one of the components $W_{1}$ or $W_{2}$ of $\mathbf{T} \backslash\{v\}$, say $z \in W_{1}$. Then $[x, z] \subset \mathbf{T} \backslash\{v\}$ by Lemma 2.3 (i). We may assume that $x$ and $z$ are so close to $v$ that $[x, z]$ contains no point in $\mathbf{V}^{n} \backslash\{v\}$. Then $[x, z] \cap \mathbf{V}^{n}=\emptyset$, and so $x$ and $z$ are contained in the same component of $\mathbf{T} \backslash \mathbf{V}^{n}$. It follows that $U=U_{1}$, and so $X=\bar{U}_{1}=\bar{U}=Z$. This shows that $X \neq Y$ are the only $n$-tiles that contain $v$. So $v$ is contained in precisely two distinct $n$-tiles. 
(vi) Each $n$-tile contains an $n$-vertex as follows from(i) and (iii), and each vertex is contained in precisely two $n$-tiles by $(\mathrm{v})$. This implies that there are at most twice as many $n$-tiles as $n$-vertices. In particular, the number of $n$-tiles is finite, because the set $\mathbf{V}^{n}$ of $n$-vertices is finite. Actually, a more careful argument shows that the number of $n$-tiles exceeds the number of $n$-vertices by exactly one, but we will not need this stronger result.

(vii) If $X^{\prime}$ is an $(n+1)$-tile, then there exists a component $W$ of $\mathbf{T} \backslash \mathbf{V}^{n+1}$ with $\bar{W}=X^{\prime}$. Since $\mathbf{V}^{n} \subset \mathbf{V}^{n+1}$, the set $W$ is a connected subset of $\mathbf{T} \backslash \mathbf{V}^{n}$ and so contained in a unique component $U$ of $\mathbf{T} \backslash \mathbf{V}^{n}$. Then $X^{\prime}$ is contained in the $n$-tile $X:=\bar{U}$, because $X^{\prime}=\bar{W} \subset \bar{U}=X$. There can be no other $n$-tile containing $X^{\prime}$, because by (i) the set $X^{\prime}$ is a subtree of $\mathbf{T}$ and hence an infinite set, but distinct $n$-tiles can have at most one point in common by (iv).

(viii) If $X$ is an $n$-tile, then $X=\bar{U}$, where $U$ is a component of $\mathbf{T} \backslash \mathbf{V}^{n}$. Since $U$ is connected, this set cannot contain isolated points. This implies that the set $U \backslash \mathbf{V}^{n+1} \subset X$ is dense in $U$ and hence also dense in $X=\bar{U}$.

If $x \in U \backslash \mathbf{V}^{n+1}$ is arbitrary, then there exists a component $W$ of $\mathbf{T} \backslash \mathbf{V}^{n+1}$ with $x \in W$. Since $W$ is a connected subset of $\mathbf{T} \backslash \mathbf{V}^{n+1} \subset$ $\mathbf{T} \backslash \mathbf{V}^{n}$, this set must be contained in a component of $\mathbf{T} \backslash \mathbf{V}^{n}$. Since $x \in U \cap W$, it follows that $W \subset U$. Then $X^{\prime}:=\bar{W}$ is an $(n+1)$-tile with $x \in X^{\prime}$ and $X^{\prime}=\bar{W} \subset \bar{U}=X$.

This shows that if we denote by $Y$ the union of all $(n+1)$-tiles $X^{\prime} \subset X$, then $Y \subset X$ contains the set $U \backslash \mathbf{V}^{n+1}$. By (vi) there are only finitely many $(n+1)$-tiles, and so $Y$ is closed. Since $U \backslash \mathbf{V}^{n+1}$ is dense in $X$ and $U \backslash \mathbf{V}^{n+1} \subset Y$, it follows that $X=Y$ as desired.

(ix) By (v) there exists precisely one $n$-tile $Y$ distinct from $X$ with $v \in Y$. We then have $\{v\}=X \cap Y$ and $v \in \partial X$ by (iv). By (viii) there exist $(n+1)$-tiles $X^{\prime} \subset X$ and $Y^{\prime} \subset Y$ with $v \in X^{\prime} \cap Y^{\prime}$. Since $X$ and $Y$ have only the point $v$ in common, it follows that $X^{\prime} \neq Y^{\prime}$ and that $Y^{\prime}$ is not a subset of $X$. Since $v \in \mathbf{V}^{n} \subset \mathbf{V}^{n+1}$ is also an $(n+1)$-vertex, $(\mathrm{v})$ implies that $X^{\prime}$ and $Y^{\prime}$ are the only $(n+1)$-tiles that contain $v$. In particular, $X^{\prime}$ is the unique $(n+1)$-tile with $v \in X^{\prime} \subset X$.

(x) Suppose that $\partial X=\{v\} \subset \mathbf{V}^{n}$. We have $X=\bar{U}$, where $U$ is a component of $\mathbf{T} \backslash \mathbf{V}^{n}$. As we have seen in the proof of (ii), there is a component $W$ of $\mathbf{T} \backslash\{v\}$ with $U \subset W$. We claim that $U=W$.

To see this, we argue by contradiction and assume that $U \neq W$. Then there exists a point $x \in U \subset W$, as well as a point $y \in W \backslash U$. Hence $[x, y] \cap \mathbf{V}^{n} \neq \emptyset$, because otherwise $y \in U$. So as we travel from $x$ to $y$ along $[x, y]$, there must be a first point $u \in[x, y]$ that belongs 
to $\mathbf{V}^{n}$. Then $[x, u) \subset U$, and so $[x, u] \subset \bar{U}=X$. By (ix) the $n$-vertex $u \in X$ is a boundary point of $X$. Hence $u \in \partial X=\{v\}$ and so $u=v$. Since $v=u \in[x, y]$, Lemma 2.3 (i) implies that $x$ and $y$ lie in different components of $\mathbf{T} \backslash\{v\}$. Since $x$ and $y$ lie in the same component $W$ of $\mathbf{T} \backslash\{v\}$, this is a contradiction. We see that $U=W$ and so $X=\bar{U}=\bar{W}$ as desired.

We now discuss some metric properties of vertices and tiles. Since $\mathbf{V}^{n}$ consists of $\delta^{n}$-separated points, for distinct $u, v \in \mathbf{V}^{n}$ we have

$$
d(u, v) \geq \delta^{n} .
$$

For each $n$-tile $X^{n}$ we have

$$
\begin{aligned}
& \operatorname{diam}\left(X^{n}\right) \asymp \delta^{n}, \text { or more precisely } \\
& \delta^{n} \leq \operatorname{diam}\left(X^{n}\right) \leq 3 \beta \delta^{n} .
\end{aligned}
$$

Indeed, the upper bound follows from Proposition 4.2.

To see that the lower bound is also true, first note that $\emptyset \neq \partial X^{n} \subset$ $\mathbf{V}^{n}$ by Lemma 5.1 (i) and (iii). If $\partial X^{n}$ is a singleton set $\{v\} \subset \mathbf{V}^{n}$, then $X^{n}$ is equal to the closure of one of the two components of $\mathbf{T} \backslash\{v\}$ by Lemma $5.1(\mathrm{x})$. Since $v$ satisfies 4.1 , we have

$$
\operatorname{diam}\left(X^{n}\right) \geq D_{\mathbf{T}}(v) \geq \beta \delta^{n} \geq \delta^{n},
$$

as desired (recall that $\beta \geq 1$ ).

If $\partial X^{n}$ contains two distinct points in $\mathbf{V}^{n}$, we obtain the lower bound in (5.3) from (5.2).

We have good separation of $n$-tiles in the following sense. If $X^{n}, Y^{n} \in$ $\mathbf{X}^{n}$ are disjoint $n$-tiles, then

$$
\operatorname{dist}\left(X^{n}, Y^{n}\right) \geq \delta^{n} .
$$

To see this, pick points $x \in X:=X^{n}$ and $y \in Y:=Y^{n}$ such that $d(x, y)=\operatorname{dist}(X, Y)$. As we travel from $x$ to $y$ along the arc $[x, y]$, we must meet the sets $\partial X$ and $\partial Y$ because $X$ and $Y$ are disjoint. Suppose $u \in[x, y] \cap \partial X$ and $v \in[x, y] \cap \partial Y$. Then $u$ and $v$ are distinct $n$-vertices and it follows that

$$
\operatorname{dist}(X, Y)=d(x, y)=\operatorname{diam}[x, y] \geq d(u, v) \geq \delta^{n},
$$

as desired.

Since each point $v \in \mathbf{V}^{n}$ is a $(\beta, \gamma)$-good double point at scale $\delta^{n}$, by (4.1) we have

$$
D_{\mathbf{T}}(v) \geq \beta \delta^{n}
$$

and so the components of $\mathbf{T} \backslash\{v\}$ are large. 
Each $n$-vertex $v$ stays away from the branch points of $\mathbf{T}$ in a controlled way. More precisely, by (4.2) for each branch point $b$ of $\mathbf{T}$ we have

$$
d(v, b) \geq \gamma \min \left\{H_{\mathbf{T}}(b), \delta^{n}\right\} .
$$

Finally, for our later discussion it is convenient to set $\mathbf{V}^{0}=\emptyset$ and regard $X^{0}:=\mathbf{T}$ as the only 0 -tile. Then $\mathbf{X}^{0}=\{\mathbf{T}\}$. Clearly (5.3) is still true.

Chains. An $n$-chain for $n \in \mathbb{N}_{0}$ is a finite non-empty sequence $P$ of $n$-tiles $X_{1}, \ldots, X_{r}$ with $X_{i} \cap X_{i+1} \neq \emptyset$ for $i=1, \ldots, r-1$. Again we call $P$ simply a chain if its level $n$ is clear from the context. We call $r \in \mathbb{N}$ the length of $P$. The chain $P$ joins the points $x, y \in \mathbf{T}$ if $x \in X_{1}$ and $y \in X_{r}$. It is simple if $X_{i} \neq X_{i+1}$ for $i=1, \ldots, r-1$ and $X_{i} \cap X_{j}=\emptyset$ for $|i-j| \geq 2$. The tiles in a simple chain $P$ are all distinct.

Given two distinct points $x, y \in \mathbf{T}$, we say that $P$ is a simple $n$-chain joining $x$ and $y$ if $P$ is simple, $X_{1}$ is the only $n$-tile in $P$ containing $x$, and $X_{r}$ is the only $n$-tile in $P$ containing $y$ (note that these requirements are stronger than saying that $P$ is simple and that $P$ joins $x$ and $y$, because the latter two conditions allow $x \in X_{1} \cap X_{2}$ ).

We use the notation $|P|:=\bigcup_{i=1}^{r} X_{i}$. We say that $P$ contains a point $x$, if $x \in|P|$. Another $n$-chain $Q$ is called a subchain of $P$ if the sequence of $n$-tiles in $Q$ is obtained by deleting some of the tiles in $P$ while keeping the order of the remaining tiles.

Lemma 5.2. Let $n \in \mathbb{N}_{0}$ and $x, y \in \mathbf{T}$ be distinct points. Then the following statements are true:

(i) There exists a unique simple $n$-chain $P$ joining $x$ and $y$.

(ii) If $P$ is the simple $n$-chain and $\widetilde{P}$ is another $n$-chain joining $x$ and $y$, then $|P| \subset|\widetilde{P}|$. More precisely, every $n$-tile in $P$ also belongs to $\widetilde{P}$.

We will often use the notation $P_{x y}^{n}$ for the unique simple $n$-chain joining the points $x, y \in \mathbf{T}, x \neq y$.

Proof of Lemma 5.2. Let $x, y \in \mathbf{T}$ with $x \neq y$ be arbitrary. We will exhibit an algorithm that produces a simple $n$-chain $P$ joining $x$ and $y$, and we will see that $P$ is the unique such $n$-chain.

Let $x<v_{1}<\cdots<v_{r-1}<y$ with $r \in \mathbb{N}$ be the distinct $n$-vertices in $(x, y)$ arranged in the natural order $<$ on $[x, y]$ (obtained by identifying $[x, y]$ with the unit interval $[0,1]$ ). This list can be empty (then $r=1$ ). We set $v_{0}:=x$ and $v_{r}:=y$. Then for $i=1, \ldots, r$ the open arc 
$\left(v_{i-1}, v_{i}\right) \subset[x, y]$ is a connected set in the complement of the set of $n$ vertices in $\mathbf{T}$. Therefore, there exists a unique $n$-tile $X_{i}$ with $\left(v_{i-1}, v_{i}\right) \subset$ $X_{i}$. Then $\left[v_{i-1}, v_{i}\right] \subset X_{i}$, because $X_{i}$ is a closed set. For $i=1, \ldots, r-1$ the $n$-vertex $v_{i}$ separates the sets $\left(v_{i-1}, v_{i}\right)$ and $\left(v_{i}, v_{i+1}\right)$, and so these sets must lie in different components of $\mathbf{T} \backslash\left\{v_{i}\right\}$ by Lemma 2.3 (i). Closures of such components can have at most the point $v_{i}$ in common as follows from Lemma 2.3 (iii), This implies that $X_{i} \cap X_{i+1}=\left\{v_{i}\right\}$. If $1 \leq i<j \leq r$ and $j-i \geq 2$, then a similar argument using a point $p \in[x, y]$ with $v_{i}<p<v_{i+1}$ shows that $X_{i} \cap X_{j}=\emptyset$. Based on this discussion one can now easily check that the $n$-tiles $X_{1}, \ldots, X_{r}$ form a simple $n$-chain $P$ joining $x$ and $y$.

Now suppose $\widetilde{P}$ is another $n$-chain joining $x$ and $y$. Then $|\widetilde{P}|$ is a path-connected set containing $x$ and $y$, and thus $[x, y] \subset|\widetilde{P}|$. In particular, $\left(v_{i-1}, v_{i}\right) \subset|\widetilde{P}|$ for $i=1, \ldots, r$. Since $X_{i}$ contains $\left(v_{i-1}, v_{i}\right)$ and all other $n$-tiles are disjoint from $\left(v_{i-1}, v_{i}\right)$ as follows from Lemma 5.1 (iv), $X_{i}$ must be one of the $n$-tiles in $\widetilde{P}$. Statement (ii) follows.

Finally, to show uniqueness of $P$, assume that $P$ is a simple $n$-chain joining $x$ and $y$. Since $x=v_{0} \in X_{1}$ and $X_{1}$ belongs to $\widetilde{P}$, the $n$-tile $X_{1}$ must be the first tile in $\widetilde{P}$. Since $X_{2} \neq X_{1}$ (in case $r \geq 2$ ) belongs to $\widetilde{P}$ and $X_{1} \cap X_{2}=\left\{v_{1}\right\} \neq \emptyset$, the $n$-tile $X_{2}$ must be the second tile in $\widetilde{P}$. Continuing in this manner, we see that the tiles in $\widetilde{P}$ are given by $X_{1}, \ldots, X_{r}$. So $\widetilde{P}=P$ and the uniqueness of $P$ follows.

We can construct simple $(n+1)$-chains from simple $n$-chains.

Lemma 5.3. Let $n \in \mathbb{N}_{0}$ and $x, y \in \mathbf{T}$ be distinct points. Suppose the simple $n$-chain $P$ joining $x$ and $y$ consists of the $n$-tiles $X_{1}, \ldots, X_{r}$, where $r \in \mathbb{N}$. For $i=1, \ldots, r-1$ let $v_{i}$ be the unique $n$-vertex in $X_{i} \cap X_{i+1}$, and let $v_{0}=x$ and $v_{r}=y$. If for $i=1, \ldots, r$ we denote by $P_{i}^{\prime}$ the simple $(n+1)$-chain joining $v_{i-1}$ and $v_{i}$, then the following statements are true:

(i) The simple $(n+1)$-chain $P^{\prime}$ joining $x$ and $y$ is obtained by concatenating $P_{1}^{\prime}, \ldots, P_{r}^{\prime}$.

(ii) The chain $P_{i}^{\prime}$ consists precisely of all $(n+1)$-tiles $X^{\prime} \subset X_{i}$ such that $X^{\prime} \cap\left(v_{i-1}, v_{i}\right) \neq \emptyset$.

Proof. As in the proof of Lemma 5.2 , let $v_{1}<\cdots<v_{r-1}$ be the distinct $n$-vertices in $(x, y)$ arranged in the natural order $<$ on $[x, y]$. Then $X_{i}$ is the unique $n$-tile that contains $\left[v_{i-1}, v_{i}\right]$ for $i=1, \ldots, r$, and we have $\left\{v_{i}\right\}=X_{i} \cap X_{i+1}$ for $i=1, \ldots, r-1$.

We can find the simple $(n+1)$-chain $P^{\prime}$ joining $x$ and $y$ by arranging the $(n+1)$-vertices in $(x, y)$ in the order $<$. Since the $n$-vertices 
$v_{1}, \ldots, v_{r-1}$ are also $(n+1)$-vertices, they will be among these $(n+1)$ vertices in $(x, y)$. This means that we may assume that all the $(n+1)$ vertices in $(x, y)$ are labeled $v_{i}^{j}$ so that

$$
\begin{aligned}
x & <v_{1}^{1}<\cdots<v_{1}^{s_{1}-1}<v_{1}^{s_{1}}=v_{1}=v_{2}^{0}<\cdots<v_{2}^{s_{2}}=v_{2} \\
& =v_{3}^{0}<\cdots<v_{r-1}^{s_{r-1}}=v_{r-1}=v_{r}^{0}<\cdots<v_{r}^{s_{r}-1}<y .
\end{aligned}
$$

Here $s_{1}, \ldots, s_{r} \in \mathbb{N}$. We set $v_{1}^{0}=x$ and $v_{r}^{s_{r}}=y$. Then the argument in the proof of Lemma 5.2 shows that for $i=1, \ldots, r$ and $j=1, \ldots, s_{i}$ there exists a unique $(n+1)$-tile $X_{i}^{j}$ such that $\left[v_{i}^{j-1}, v_{i}^{j}\right] \subset X_{i}^{j}$. Moreover, the simple $(n+1)$-chain $P^{\prime}$ joining $x$ and $y$ is given by

$$
X_{1}^{1}, \ldots, X_{1}^{s_{1}}, X_{2}^{1}, \ldots, X_{2}^{s_{2}}, \ldots, X_{r}^{1}, \ldots, X_{r}^{s_{r}} .
$$

It is also clear that for $i=1, \ldots, r$ the simple $(n+1)$-chain $P_{i}^{\prime}$ joining $v_{i-1}=v_{i}^{0}$ and $v_{i}=v_{i}^{s_{i}}$ is given by $X_{i}^{1}, \ldots, X_{i}^{s_{i}}$, because $v_{i}^{1}<\cdots<v_{i}^{s_{i}-1}$ are all the $(n+1)$-vertices in $\left(v_{i-1}, v_{i}\right)=\left(v_{i}^{0}, v_{i}^{s_{i}}\right) \subset(x, y)$. This implies that $P^{\prime}$ is the concatenation of the chains $P_{1}^{\prime}, \ldots, P_{r}^{\prime}$. Statement (i) follows.

To see (ii), we fix $i \in\{1, \ldots, r\}$. Let $X_{i}^{j}$ with $j \in\left\{1, \ldots, s_{i}\right\}$ be an $(n+1)$-tile from the simple $(n+1)$-chain $P_{i}^{\prime}$ joining $v_{i-1}$ and $v_{i}$. Then $X_{i}^{j}$ is contained in a unique $n$-tile by Lemma 5.1 (vii). Note that $\left(v_{i}^{j-1}, v_{i}^{j}\right) \subset X_{i}^{j}$ and $\left(v_{i}^{j-1}, v_{i}^{j}\right) \subset\left(v_{i-1}, v_{i}\right)$; so $X_{i}^{j}$ contains points in $\left(v_{i-1}, v_{i}\right)$. Since $\left(v_{i-1}, v_{i}\right) \subset X_{i}$ and $\left(v_{i-1}, v_{i}\right)$ contains no $n$-vertices, Lemma 5.1 (iv) implies all $n$-tiles except $X_{i}$ are disjoint from $\left(v_{i-1}, v_{i}\right)$. We conclude that $X_{i}^{j} \subset X_{i}$. This shows that $P_{i}^{\prime}$ consists of $(n+1)$-tiles that are contained in $X_{i}$ and meet $\left(v_{i-1}, v_{i}\right)$.

Conversely, suppose $Z \subset X_{i}$ is an $(n+1)$-tile with $Z \cap\left(v_{i-1}, v_{i}\right) \neq \emptyset$. Since

$$
\left(v_{i-1}, v_{i}\right)=\left(v_{i}^{0}, v_{i}^{1}\right) \cup\left\{v_{i}^{1}\right\} \cup\left(v_{i}^{1}, v_{i}^{2}\right) \cup \cdots \cup\left\{v_{i}^{s_{i}-1}\right\} \cup\left(v_{i}^{s_{i}-1}, v_{i}^{s_{i}}\right),
$$

we then have $Z \cap\left(v_{i}^{j-1}, v_{i}^{j}\right) \neq \emptyset$ for some $j \in\left\{1, \ldots, s_{i}\right\}$ or $v_{i}^{j} \in Z$ for some $j \in\left\{1, \ldots, s_{i}-1\right\}$.

In the first case, $Z=X_{i}^{j}$, because no $(n+1)$-tile except $X_{i}^{j} \supset$ $\left(v_{i}^{j-1}, v_{i}^{j}\right)$ contains points in $\left(v_{i}^{j-1}, v_{i}^{j}\right)$. This follows from Lemma 5.1 (iv) because no point in $\left(v_{i}^{j-1}, v_{i}^{j}\right)$ is an $(n+1)$-vertex.

In the second case, when $v_{i}^{j} \in Z$ for some $j \in\left\{1, \ldots, s_{i}-1\right\}$, we have $Z=X_{i}^{j}$ or $Z=X_{i}^{j+1}$, because $X_{i}^{j}$ and $X_{i}^{j+1}$ are the only $(n+1)$-tiles that contain the $(n+1)$-vertex $v_{i}^{j}$ (this follows from Lemma 5.1 (v)).

In any case, $Z$ is one of the tiles in the chain $P_{i}^{\prime}$, and statement (ii) follows. 
Choosing $\delta$. We now are going to choose the parameter $\delta>0$ used in the definition of vertices and tiles small enough so that $(n+1)$-tiles are contained in $n$-tiles in a "controlled way". The choice of $\delta$ will depend on the constants $\beta$ and $\gamma$ fixed at the beginning of this section. Recall that we imposed the preliminary condition $0<\delta<1 /(3 \beta)$ for the definition of tiles and vertices. The ultimate choice of $\delta$ will be discussed after the proof of Lemma 5.5.

Lemma 5.4. If $0<\delta<1 /(3 \beta)$ is sufficiently small only depending on the doubling constant $N$ of $\mathbf{T}$, then the following statements are true for all $n \in \mathbb{N}_{0}$ :

(i) Each n-tile $X$ contains at least three $(n+1)$-tiles.

(ii) If $u$ and $v$ are distinct $n$-vertices, then the simple $(n+1)$-chain joining $u$ and $v$ has length $\geq 3$.

It follows from the first statement that then there are least three 1-tiles. The second statement implies that each $(n+1)$-tile $X^{\prime}$ contains at most one $n$-vertex.

Proof. Fix $n \in \mathbb{N}_{0}$. Then we know by (5.3) that $\operatorname{diam}(X) \geq \delta^{n}$ for each $n$-tile $X$, and $\operatorname{diam}\left(X^{\prime}\right) \leq 3 \beta \delta^{n+1}$ for each $(n+1)$-tile $X^{\prime}$. It follows that (i) is true for $0<\delta<1 /(6 \beta)$.

If $u$ and $v$ are distinct $n$-vertices, then $d(u, v) \geq \delta^{n}$ by (5.2). Again we have $\operatorname{diam}\left(X^{\prime}\right) \leq 3 \beta \delta^{n+1}$ for each $(n+1)$-tile $X^{\prime}$. Thus (ii) is also true if $0<\delta<1 /(6 \beta)$.

In the next lemma we consider the location of $(n+1)$-vertices in an $n$-tile. In the proof we will invoke (5.6) derived from (4.2). This is the ultimate reason why we want the elements in $\mathbf{V}^{n}$ to satisfy 4.2 in addition to (4.1) (with $\Delta=\delta^{n}$ ). A consequence will be the subsequent Lemma 5.6. It guarantees that if we decompose an $n$-tile $X$ into $(n+1)$ tiles, then a simple chain of $(n+1)$-tiles joining two distinct points in $\partial X$ does not encounter other points in $\partial X$. This in turn is behind the important estimate in Lemma 6.1 (ii). It prevents blow up of the auxiliary distance functions $\varrho_{n}$ as $n \rightarrow \infty$ that we will use to define our desired geodesic metric $\varrho$ on $\mathbf{T}$ and ultimately leads to the existence of the limit in (1.1).

Lemma 5.5. If $0<\delta<1 /(3 \beta)$ is sufficiently small only depending on the doubling constant $N$ of $\mathbf{T}$, then the following statement is true. Let $n \in \mathbb{N}, X$ be an n-tile, $u \in \partial X \subset \mathbf{V}^{n}$, and $X^{\prime} \subset X$ be the unique $(n+1)$-tile with $u \in X^{\prime}$. Then there exists an $(n+1)$-vertex $u^{\prime} \in \partial X^{\prime} \backslash\{u\}$ such that $\left[u, u^{\prime}\right] \subset[u, v]$ for all $v \in \partial X \backslash\{u\}$. 


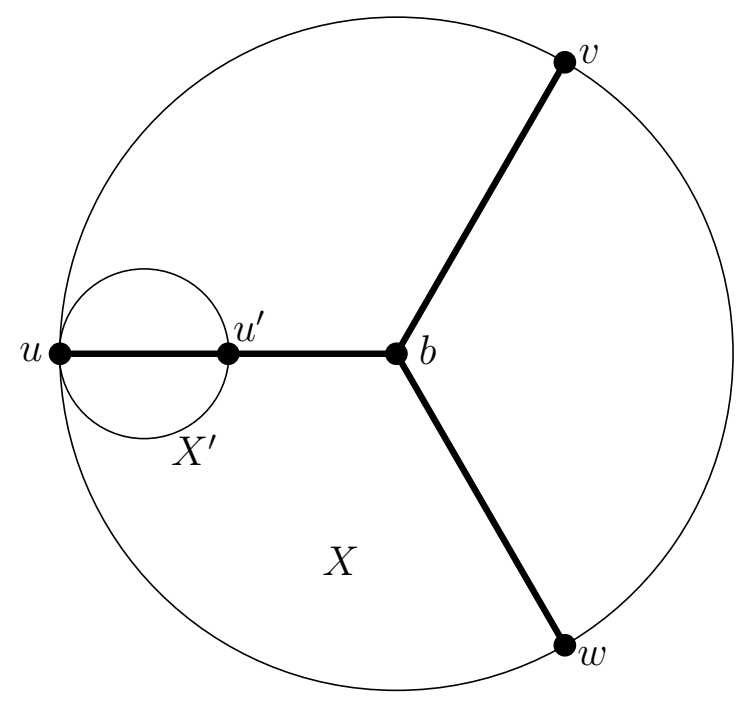

FiguRE 5. Branching in an $n$-tile.

Note that the existence of a unique $(n+1)$-tile $X^{\prime} \subset X$ with $u \in X^{\prime}$ is guaranteed by Lemma 5.1 (ix). If we are in the setting of Lemma 5.5 and $\delta$ is so small that Lemma 5.4 (ii) applies, then, as we travel from $u$ to $v$ along $[u, v] \subset X$, we must exit $X^{\prime}$, because $X^{\prime}$ contains $u$, but not $v$. So there is a last point on $[u, v]$ that belongs to $X^{\prime}$. This must be the point $u^{\prime} \in \partial X^{\prime} \backslash\{u\}$ in the statement, because $\left[u, u^{\prime}\right] \subset X^{\prime}$ and $u, v$ lie in different components of $\mathbf{T} \backslash\left\{u^{\prime}\right\}$; so $X^{\prime} \cap\left(u^{\prime}, v\right]=\emptyset$ by Lemma 5.1(ii), According to Lemma 5.5, this last point $u^{\prime}$ in $[u, v] \cap X^{\prime}$ is independent of $v$, and so we always exit $X^{\prime}$ at the same $(n+1)$-vertex $u^{\prime}$ when traveling from $u$ to any other point in $\partial X$. We will later call $u$ and $u^{\prime}$ the "main vertices" of $X^{\prime}$.

Proof of Lemma 5.5. We may assume that $\delta$ is so small that the statements in Lemma 5.4 are true. It follows from the preceding discussion, that if $v \in \partial X \backslash\{u\}$, then there is a last point $u^{\prime} \in[u, v] \cap X^{\prime}$ as we travel from $u$ to $v$ along $[u, v]$. Clearly, $u^{\prime} \in \partial X^{\prime} \subset \mathbf{V}^{n+1}$. We also have $u \neq u^{\prime}$, because if $u=u^{\prime}$, then $(u, v] \subset X$ is disjoint from $X^{\prime}$ and so the set $(u, v]$ would be covered by the finitely many $(n+1)$-tiles $Y \subset X$ distinct from $X^{\prime}$. These tiles $Y$ then also cover $[u, v]$, and so $u$ is contained in a tile $Y \subset X$ distinct from $X^{\prime}$. We know that this is impossible, and so indeed $u^{\prime} \neq u$.

It remains to show that this last point $u^{\prime}$ on $[u, v] \cap X^{\prime}$ is independent of $v$. So suppose $w \neq v$ is another vertex in $\partial X \backslash\{u\}$. Then the points $u, v, w \in \partial X$ are distinct. For an illustration of the ensuing argument see Figure 5 . 
Since $\mathbf{T}$ is a tree, the arcs $[u, v]$ and $[u, w]$ share an initial segment $[u, b]=[u, v] \cap[u, w]$, where $b \in \mathbf{T}$, but no other points. It suffices to show that $\left[u, u^{\prime}\right] \subset[u, b]$. Since both points $u^{\prime}$ and $b$ lie on $[u, v]$, we have $\left[u, u^{\prime}\right] \subset[u, b]$ or $[u, b] \subset\left[u, u^{\prime}\right]$. The first alternative is necessarily true if we can show that diam $\left[u, u^{\prime}\right]<\operatorname{diam}[u, b]$.

First note that $b \neq u$. Indeed, if $b=u$ then $u \in[v, w]=[v, u] \cup$ $[u, w]$, and so $v$ and $w$ would lie in distinct components of $\mathbf{T} \backslash\{u\}$. By Lemma 5.1 (ii) this is impossible, because $v$ and $w$ lie in the same tile $X$. Similarly, $b \neq v$ and $b \neq w$.

It follows from Lemma 2.4 that $b$ is a branch point of $\mathbf{T}$ and $u, v, w$ lie in distinct components $U_{u}, U_{v}, U_{w}$ of $\mathbf{T} \backslash\{b\}$, respectively. Note that $U_{u}$ contains one component $V_{u}$ of $\mathbf{T} \backslash\{u\}$. Thus by (5.5),

$$
\operatorname{diam}\left(U_{u}\right) \geq \operatorname{diam}\left(V_{u}\right) \geq D_{\mathbf{T}}(u) \geq \beta \delta^{n} .
$$

Similarly, $\operatorname{diam}\left(U_{v}\right) \geq \beta \delta^{n}$ and $\operatorname{diam}\left(U_{w}\right) \geq \beta \delta^{n}$. It follows that

$$
H_{\mathbf{T}}(b) \geq \min \left\{\operatorname{diam}\left(U_{u}\right), \operatorname{diam}\left(U_{v}\right), \operatorname{diam}\left(U_{w}\right)\right\} \geq \beta \delta^{n} \geq \delta^{n},
$$

since $\beta \geq 1$. Thus by 5.6 we have

$$
d(u, b) \geq \gamma \min \left\{H_{\mathbf{T}}(b), \delta^{n}\right\} \geq \gamma \delta^{n} .
$$

By (5.3) we know that $\operatorname{diam}\left(X^{\prime}\right) \leq 3 \beta \delta^{n+1}$, and so

$$
d\left(u, u^{\prime}\right) \leq \operatorname{diam}\left(X^{\prime}\right) \leq 3 \beta \delta^{n+1} \text {. }
$$

So if we assume that $0<\delta<\gamma /(3 \beta)$, then it follows that

$$
\operatorname{diam}\left[u, u^{\prime}\right]=d\left(u, u^{\prime}\right) \leq 3 \beta \delta^{n+1}<\gamma \delta^{n} \leq d(u, b)=\operatorname{diam}[u, b] .
$$

As we have seen, this implies $\left[u, u^{\prime}\right] \subset[u, b]$ as desired.

For the rest of the paper we fix $0<\delta<1 /(3 \beta)$ such that the statements of Lemma 5.4 and Lemma 5.5 are true. As we see from the proofs, it is enough to choose $\delta=\frac{1}{2} \min \{1 /(6 \beta), \gamma /(3 \beta)\}$. Then $\delta$ only depends on the doubling constant $N$ of $\mathbf{T}$, because this is true for $\beta$ and $\gamma$. The sets $\mathbf{V}^{n}$ of vertices and $\mathbf{X}^{n}$ of tiles for $n \in \mathbb{N}_{0}$ as constructed in the beginning of this section correspond to this choice of $\delta$ and will be fixed from now on.

Let us record some consequences.

Lemma 5.6. Let $n \in \mathbb{N}_{0}, X$ be an $n$-tile, and $u, v \in \partial X \subset \mathbf{V}^{n}$ with $u \neq v$. Then the simple $(n+1)$-chain $P_{u v}^{n+1}$ joining $u$ to $v$ consists precisely of all $(n+1)$-tiles $X^{\prime} \subset X$ with $X^{\prime} \cap[u, v] \neq \emptyset$. Moreover, $P_{u v}^{n+1}$ does not contain any point $w \in \partial X$ distinct from $u$ and $v$.

It follows from the definition of $P_{u v}^{n+1}$ that only the first tile of $P_{u v}^{n+1}$ contains $u$, and only the last tile contains $v$. So $P_{u v}^{n+1}$ has "contact" 
with $\partial X$ only twice: in its first tile, where it meets $u$, and in its last tile, where it meets $v$.

Proof of Lemma 5.6. Let $P:=P_{u v}^{n+1}$, and assume $P$ is given by the $(n+1)$-tiles $X_{1}, \ldots, X_{r}$, where $r \in \mathbb{N}$.

The first statement follows from considerations similar to the ones in the proof of Lemma 5.3 (ii)). Note that Lemma 5.1 (ix) implies that $X^{\prime}=X_{1}$ is the only $(n+1)$-tile $X^{\prime} \subset X$ with $u \in X^{\prime}$, and $X^{\prime}=X_{r}$ is the only $(n+1)$-tile $X^{\prime} \subset X$ with $v \in X^{\prime}$.

To prove the second statement, we argue by contradiction and assume that it is false. Then there exists a point $w \in|P| \cap \partial X$ that is distinct from $u$ and $v$. Then $w \in X_{i}$ for some $i \in\{1, \ldots, r\}$. In fact, since an $(n+1)$-tile cannot contain two distinct $n$-vertices (see Lemma 5.4 (ii)), we have $2 \leq i \leq r-1$. Let $v_{i-1}$ and $v_{i}$ be the (unique) points in $X_{i-1} \cap X_{i}$ and $X_{i} \cap X_{i+1}$, respectively.

We now choose the $(n+1)$-vertex $w^{\prime} \in \partial X_{i}$ for the $n$-vertex $w \in$ $X_{i} \subset X$ as in Lemma 5.5. In particular, $w^{\prime}$ is the last point on both $[w, u]$ and $[w, v]$ as we travel from $w$ to $u$ or from $w$ to $v$.

Since the set $X_{1} \cup \cdots \cup X_{i}$ is connected, we have $[w, u] \subset X_{1} \cup \cdots \cup X_{i}$. So the last point $w^{\prime}$ in $[w, u] \cap X_{i}$ must be a point in $X_{1} \cup \cdots \cup X_{i-1}$. Since $P$ is the simple $(n+1)$-chain joining $u$ and $v$, this is only possible if $w^{\prime}=v_{i-1}$, because there is no other common point of $X_{i}$ with any of the tiles $X_{1}, \ldots, X_{i-1}$. Similarly, by considering $[w, v] \subset X_{i} \cup \cdots \cup X_{r}$, we see that $w^{\prime}=v_{i}$. This is impossible, because then $w^{\prime} \in X_{i-1} \cap X_{i+1} \neq \emptyset$, contradicting the fact that $P$ is a simple $(n+1)$-chain.

The following statement gives uniform control for the local combinatorics of tiles.

Lemma 5.7. There is a constant $K \in \mathbb{N}$ such that the following statements are true for each $n \in \mathbb{N}_{0}$ and each $n$-tile $X$ :

(i) There are at most $K n$-tiles that intersect $X$.

(ii) There are at most $K(n+1)$-tiles contained in $X$.

Proof. (i) Let $X_{1}, \ldots, X_{k}$ denote all the $n$-tiles distinct from $X$ that intersect $X$, where $k \in \mathbb{N}_{0}$ (if $n=0$, we have $k=0$, and this list is empty). Since diameters of $n$-tiles are comparable to $\delta^{n}$ as in (5.3), there is a constant $C=C(N)>0$ only depending on the doubling constant $N$ of $\mathbf{T}$ (and hence independent of $n$ and $X$ ) such that

$$
X \cup X_{1} \cup \cdots \cup X_{k} \subset B\left(x, C \delta^{n}\right),
$$

where $x$ is some point in $X$.

For $i=1, \ldots, k$ the $n$-tiles $X$ and $X_{i}$ intersect in an $n$-vertex $v_{i}$ (see Lemma 5.1 (iv)). By Lemma 5.1 (v) each of these $n$-vertices $v_{i}$ is 
contained in precisely two $n$-tiles, namely $X$ and $X_{i}$. It follows that $v_{i} \neq v_{j}$ for $i \neq j$. Thus $B\left(x, C \delta^{n}\right) \supset X \cup X_{1} \cup \cdots \cup X_{k}$ contains at least $k$ distinct $n$-vertices $v_{1}, \ldots, v_{k}$. Since $C$ only depends on $N$ and the $n$-vertices $v_{1}, \ldots, v_{k}$ are $\delta^{n}$-separated by (5.2), it follows that there is a constant $K_{1}=K_{1}(N) \in \mathbb{N}$ such that $k \leq K_{1}$.

(ii) As before, there is a constant $C=C(N)>0$ independent of $n$ and $X$ such that $X \subset B\left(x, C \delta^{n}\right)$, where $x$ is some point in $X$ (see $(5.3)$ ). If $k \in \mathbb{N}$ is the number of $(n+1)$-tiles contained in $X$, then $X$ also contains at least $k / 2$ distinct $(n+1)$-vertices, because each $(n+1)$-tile contains at least one $(n+1)$-vertex and each $(n+1)$-vertex is contained in at most two $(n+1)$-tiles. These $(n+1)$-vertices are $\delta^{n+1}$-separated by $(5.2)$. So it follows that there is a constant $K_{2} \in \mathbb{N}$ only depending on $C$ and $\delta$ (and hence independent of $n$ and $X$ ) such that $k \leq K_{2}$.

If we now choose $K:=\max \left\{K_{1}, K_{2}\right\}$, then statements (i) and (ii) are both true for all $n \in \mathbb{N}_{0}$ and all $n$-tiles $X$.

\section{Weights AND MAIN VERTiCES OF TILES}

We will now define weights of tiles. Later they will be used to construct our desired geodesic metric $\varrho$. The weight of each $n$-tile $X$, $n \in \mathbb{N}_{0}$, is a number $w(X) \in(0, \infty)$. We will define it by an inductive process over the level $n \in \mathbb{N}_{0}$.

Once we have determined weights of tiles, we can define the $w$-length of an $n$-chain $P$ given by the $n$-tiles $X_{1}, \ldots, X_{r}$ as

$$
\operatorname{length}_{w}(P):=\sum_{i=1}^{r} w\left(X_{i}\right) .
$$

For the construction of the geodesic metric it is desirable to have a relation between the weight $w(X)$ of an $n$-tile $X$ and the $w$-length of some simple $(n+1)$-chains $P$ joining points on the boundary of $X$. For this reason, we will single out two distinct points $p, q \in \partial X$ (i.e., two $n$-vertices in $X)$ as the main vertices of $X$. Of course, this requires that $\# \partial X \geq 2$. In this case, we call $X$ an arc-tile, because we think of $X$ as carrying the distinguished arc $[p, q]$. Otherwise, $\# \partial X \leq 1$. If $\# \partial X=1$, then we call $X$ a leaf-tile. Finally, if $\partial X=\emptyset$, then necessarily $n=0$ and $X=\mathbf{T}$ (this follows from Lemma 5.1 (iii)].

If $X$ is an arc-tile, $p, q \in \partial X$ are the main vertices of $X$, and $P=$ $P_{p q}^{n+1}$ is the unique simple $(n+1)$-chain joining $p$ and $q$, then we will choose weights in such a way that $\operatorname{length}_{w}(P)=w(X)$ (see (6.6)). This will ensure that the distance functions $\varrho_{n}$ that we use to define 
the desired geodesic metric do not degenerate as $n \rightarrow \infty$ (see $\sqrt{7.1}$ ) and Lemma 7.4).

The $(n+1)$-tiles $X^{\prime} \subset X$ that do not intersect $[p, q]$ will be given a uniformly small relative weight $\epsilon_{0}=w\left(X^{\prime}\right) / w(X)$ (see (6.7)). As a consequence, the distance functions $\varrho_{n}$ are "almost" decreasing (Lemma 7.2) and have a limit as $n \rightarrow \infty$ (Lemma 7.3). Letting $\epsilon_{0} \rightarrow 0$ will later also allow us to derive Theorem 1.3 .

After this outline of some of the ideas, we will now give the details for the definition of weights and main vertices of tiles. Let $K \in \mathbb{N}$ be the constant from Lemma 5.7. We fix a parameter

$$
0<\epsilon_{0} \leq 1 /(3 K)
$$

There is a single 0 -tile $X^{0}=\mathbf{T}$. We set $w\left(X^{0}\right):=1$. Since $\partial X^{0}=\emptyset$, we do not define main vertices of $X^{0}$.

We now assume that for some $n \in \mathbb{N}_{0}$ we have defined the weight of each $n$-tile $X$ and its main vertices if $\# \partial X \geq 2$. We fix $X$ and want to define weights of $(n+1)$-tiles $X^{\prime} \subset X$ and main vertices for arc-tiles $X^{\prime}$. Since every $(n+1)$-tile is contained in a unique $n$-tile (see Lemma 5.1 (vii)), this will provide the necessary inductive step. Figure 6 illustrates how we will choose weights and main vertices of $(n+1)$-tiles $X^{\prime} \subset X$ in the ensuing discussion. In this figure, we indicated relative weights $w\left(X^{\prime}\right) / w(X)$.

Assume first that $\partial X=\emptyset$. This happens precisely when $n=0$ and $X=X^{0}=\mathbf{T}$. We set $w\left(X^{\prime}\right):=\epsilon_{0} w(X)=\epsilon_{0}$ for each 1-tile $X^{\prime}$. If $X^{\prime}$ is an arc-tile and so $\# \partial X^{\prime} \geq 2$, we pick two (arbitrary) distinct points in $\partial X^{\prime}$ and declare them to be the main vertices of $X^{\prime}$.

Suppose now that $X$ is a leaf-tile, i.e., $\# \partial X=1$. We then set $w\left(X^{\prime}\right)=\epsilon_{0} w(X)$ for each $(n+1)$-tile $X^{\prime} \subset X$.

To define main vertices of $(n+1)$-tiles that are arc-tiles contained in $X$, recall first that there is a unique $(n+1)$-tile $X^{\prime} \subset X$ that contains the (only) $n$-vertex $u \in \partial X$. It follows from our choice of $\delta$ and Lemma 5.4 (i) that $X^{\prime}$ must be an arc-tile. We declare $u$ and some other (arbitrary) $(n+1)$-vertex $u^{\prime} \in \partial X^{\prime}$ with $u^{\prime} \neq u$ to be the main vertices of $X^{\prime}$.

If an $(n+1)$-tile $X^{\prime} \subset X$ is an arc-tile and does not intersect $\partial X$, we again declare two arbitrary distinct $(n+1)$-vertices in $\partial X^{\prime}$ to be the main vertices of $X^{\prime}$. This completes the inductive step in the case that $X$ is a leaf-tile.

Finally, suppose that $X$ is an arc-tile, i.e., $\# \partial X \geq 2$, and let $p, q \in$ $\partial X$ be the main vertices of $X$. By Lemma 5.1 (ix) there are unique $(n+1)$-tiles $X_{p}^{\prime} \subset X$ and $X_{q}^{\prime} \subset X$ containing $p$ and $q$, respectively. By our choice of $\delta$ and Lemma 5.4 (ii), the tiles $X_{p}^{\prime}$ and $X_{q}^{\prime}$ are distinct 


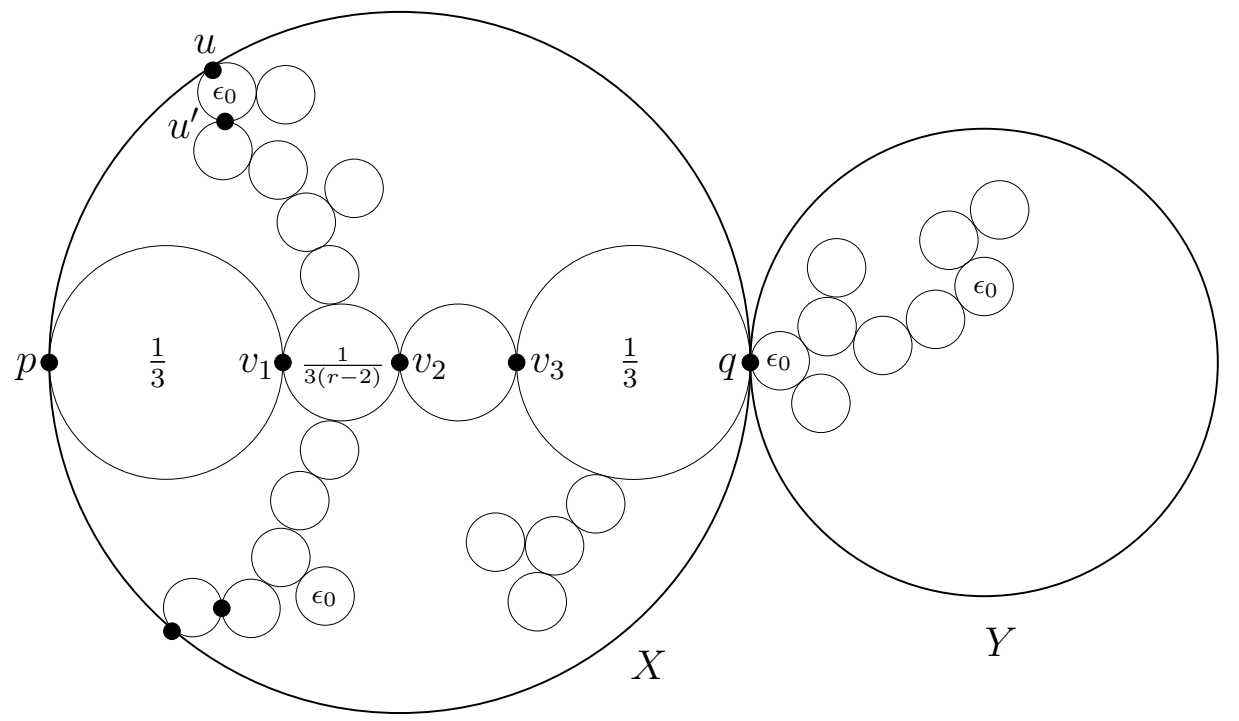

FiguRE 6. Relative weights and main vertices of tiles

and disjoint. We set

$$
w\left(X_{p}^{\prime}\right)=w\left(X_{q}^{\prime}\right):=\frac{1}{3} w(X) .
$$

Suppose the simple $(n+1)$-chain $P:=P_{p q}^{n+1}$ joining $p$ and $q$ is given by the $(n+1)$-tiles

$$
X_{1}^{\prime}=X_{p}^{\prime}, X_{2}^{\prime}, \ldots, X_{r}^{\prime}=X_{q}^{\prime} .
$$

Then $P$ consists of tiles $X_{i}^{\prime}$ contained in $X$. Since $p \neq q$ we have $r \geq 3$. Moreover, $r \leq K$ by Lemma 5.7 (ii). Note that $P=P_{p q}^{n+1}$ consists precisely of all tiles $X^{\prime} \subset X$ with $X^{\prime} \cap[p, q] \neq \emptyset$ (see Lemma 5.6).

We have $X_{1}^{\prime}=X_{p}^{\prime}$ and $X_{r}^{\prime}=X_{q}^{\prime}$ and so the weights $w\left(X_{1}^{\prime}\right)$ and $w\left(X_{r}^{\prime}\right)$ are already defined. We set

$$
w\left(X_{i}^{\prime}\right):=\frac{1}{3(r-2)} w(X) .
$$

for $i=2, \ldots, r-1$. Then

$$
\sum_{i=1}^{r} w\left(X_{i}^{\prime}\right)=w(X) .
$$

So the weights are defined in such a way that the $w$-length (as in (6.1)) of the simple $(n+1)$-chain $P$ joining the two main vertices $p$ and $q$ of the $n$-tile $X$ is exactly equal to $w(X)$.

To define the main vertices of the tiles $X_{i}^{\prime}$, let $v_{i}$ be the (unique) point in $X_{i}^{\prime} \cap X_{i+1}^{\prime}$ for $i=1, \ldots, r-1$. Furthermore, let $v_{0}:=p$ and $v_{r}:=q$. Then $v_{i-1}$ and $v_{i}$ are distinct $(n+1)$-vertices in $\partial X_{i}^{\prime}$ for $i=1, \ldots, r$. 
We declare them to be the main vertices of $X_{i}^{\prime}$. In other words, two successive $(n+1)$-vertices on $[p, q]$ are the main vertices of the unique $(n+1)$-tile $X^{\prime} \subset X$ that contains them.

We now consider an $(n+1)$-tile $X^{\prime} \subset X$ that does not intersect $[p, q]$. We set

$$
w\left(X^{\prime}\right):=\epsilon_{0} w(X) .
$$

It remains to define the main vertices of $X^{\prime}$ if $X^{\prime}$ is an arc-tile. If $X^{\prime}$ contains a point $u \in \partial X$, we let $u^{\prime} \in \partial X^{\prime} \backslash\{u\}$ be the $(n+1)$-vertex given by Lemma 5.5. We declare $u$ and $u^{\prime}$ to be the man vertices of $X^{\prime}$. If $X^{\prime} \cap \partial X=\emptyset$, we declare two arbitrary points in $\partial X^{\prime}$ to be the main vertices of $X^{\prime}$. This concludes the definition of weights and main vertices of tiles $X^{\prime} \subset X$ in case $X$ is an arc-tile.

The inductive step is now complete, because we covered all possibilities for $X$. Therefore, weights are defined for all tiles and main vertices for all arc-tiles. To avoid possible confusion, we point out that if an $n$-vertex $u$ is contained in two distinct $n$-tiles $X$ and $Y$, and $u$ is a main vertex of $X$, then it is not necessarily a main vertex of $Y$.

The choice of relative weights and main vertices is illustrated in Figure 6. Main vertices of $(n+1)$-tiles that intersect neither $[p, q]$ nor $\partial X$ were chosen arbitrarily, and are not shown in the picture.

Note that for $r$ in 6.5 we have $3 \leq r \leq K$. Hence

$$
\epsilon_{0} \leq \frac{1}{3 K} \leq \frac{1}{3(r-2)} \leq \frac{1}{3}
$$

This and the definition of the weights imply that for each $(n+1)$-tile $X^{\prime}$ contained in an $n$-tile $X$ we have

$$
\epsilon_{0} w(X) \leq w\left(X^{\prime}\right) \leq \frac{1}{3} w(X) .
$$

Having defined the weights of tiles, we can now estimate the $w$-length of chains (see (6.1) for the definition).

Lemma 6.1. Let $n \in \mathbb{N}_{0}$ and $X$ be an $n$-tile. Then the following statements are true:

(i) For each simple $(n+1)$-chain $P$ consisting of tiles contained in $X$ we have

$$
\epsilon_{0} w(X) \leq \operatorname{length}_{w}(P) \leq \frac{4}{3} w(X) .
$$

(ii) If $X$ is an arc-tile, $u, v \in \partial X$ with $u \neq v$, and $P=P_{u v}^{n+1}$ is the simple $(n+1)$-chain joining $u$ and $v$, then

$$
\operatorname{length}_{w}(P) \leq w(X) .
$$

Here we have equality if $u$ and $v$ are the main vertices of $X$. 
Proof. (i) If $P$ is a simple $(n+1)$-chain as in the statement, then each $(n+1)$-tile $X^{\prime} \subset X$ can appear only once in $P$. Recall that the number of $(n+1)$-tiles $X^{\prime} \subset X$ is at most $K$; so there are at most $K$ such tiles $X^{\prime}$ with $w\left(X^{\prime}\right)=\epsilon_{0} w(X)$. If $X$ is an arc-tile, then among the $(n+1)$-tiles with $X^{\prime} \subset X$ there are two with $w\left(X^{\prime}\right)=\frac{1}{3} w(X)$ and an additional $r-2$ with $w\left(X^{\prime}\right)=\frac{1}{3(r-2)} w(X)$. Here $r$ is as in 6.5). Since $\epsilon_{0} K \leq \frac{1}{3}$ by our choice of $\epsilon_{0}$, we conclude that

$$
\epsilon_{0} w(X) \leq \operatorname{length}_{w}(P) \leq\left(K \epsilon_{0}+\frac{2}{3}+\frac{r-2}{3(r-2)}\right) w(X) \leq \frac{4}{3} w(X),
$$

and (i) follows.

(ii) If $P$ is the simple $(n+1)$-chain in $X$ joining the two main vertices $p$ and $q$ of $X$, then we know that $\operatorname{length}_{w}(P)=w(X)$ (see 6.6)).

Suppose the simple $(n+1)$-chain $P$ joins two distinct $n$-vertices $u, v \in$ $\partial X$, but not both $u$ and $v$ are main vertices of $X$. Lemma 5.6 then implies that at least one of the two $(n+1)$-tiles $X^{\prime} \subset X$ with $w\left(X^{\prime}\right)=$ $\frac{1}{3} w(X)$ that contain main vertices of $X$ does not belong to $P$. We conclude that

$$
\operatorname{length}_{w}(P) \leq\left(\frac{1}{3}+\frac{r-2}{3(r-2)}+K \epsilon_{0}\right) w(X) \leq w(X)
$$

Statement (ii) follows.

It is in fact possible that for a simple $(n+1)$-chain $P$ as in Lemma 6.1](i) we have $\operatorname{length}_{w}(P)>w(X)$. An example can be obtained from Figure 6, where one can find a simple $(n+1)$-chain $P$ that consists of $(n+1)$-tiles $X^{\prime} \subset X$ and contains $P_{p q}^{n+1}$ (i.e., the simple $(n+1)$-chain joining the main vertices $p$ and $q$ of $X$ ) as a proper subchain.

Let $X$ be an $n$-tile, and $v \in \partial X$. Then $v$ is an $n$-vertex, and so an $(n+1)$-vertex as well. If $X^{\prime} \subset X$ is the unique $(n+1)$-tile containing $v$, then $X^{\prime}$ is an arc-tile by our construction, and $v$ is one of the main vertices of $X^{\prime}$. Repeating this argument, we see that for each $k \geq n+1$, $v$ is a main vertex of the $k$-tile $X^{k} \subset X^{\prime}$ containing $v$, and so (6.3) implies that $w\left(X^{k}\right)=3^{-k+n+1} w\left(X^{\prime}\right)$.

Lemma 6.2. Let $n, m \in \mathbb{N}_{0}$ with $|n-m| \leq 1$. Suppose $X$ is an $n$-tile, $Y$ is an $m$-tile, and $X \cap Y \neq \emptyset$. Then

$$
w(X) \asymp w(Y),
$$

where $C(\asymp)$ is independent of $n, m, X$, and $Y$. 
Proof. We first consider the case $n=m$. If $X=Y$ there is nothing to prove, and so we assume that $X \neq Y$. There are unique tiles

$$
\begin{aligned}
& X^{0} \supset X^{1} \supset \cdots \supset X^{n}=X \\
& Y^{0} \supset Y^{1} \supset \cdots \supset Y^{n}=Y,
\end{aligned}
$$

where $X^{i}$ and $Y^{i}$ are $i$-tiles for $i=0, \ldots, n$. Let $k \leq n-1$ be the largest number such that $X^{k}=Y^{k}$. Such a number $k$ exists, since there is only a single 0-tile $X^{0}=Y^{0}=\mathbf{T}$. Then $w\left(X^{k}\right)=w\left(Y^{k}\right)$. Since $X^{k+1}, Y^{k+1} \subset X^{k}=Y^{k}$, we have $w\left(X^{k+1}\right) \asymp w\left(Y^{k+1}\right)$ with $C(\asymp)=1 /\left(3 \epsilon_{0}\right)$, as follows from $(6.8)$.

If $k+1=n$, we are done. Otherwise, if $k+1<n$, we can again apply 6.8 and obtain

$$
w\left(X^{k+2}\right) \asymp w\left(Y^{k+2}\right),
$$

where $C(\asymp)=1 /\left(3 \epsilon_{0}\right)^{2}$. Since $X^{k+1} \cap Y^{k+1} \supset X \cap Y \neq \emptyset$ and $X^{k+1} \neq$ $Y^{k+1}$, there exists a unique $(k+1)$-vertex $v$ such that $X^{k+1} \cap Y^{k+1}=$ $\{v\}$. Then $X^{k+2+i} \cap Y^{k+2+i}=\{v\}$, the point $v$ is a main vertex of $X^{k+2+i}$ and of $Y^{k+2+i}$, and so

$$
w\left(X^{k+2+i}\right)=3^{-i} w\left(X^{k+2}\right) \text { and } w\left(Y^{k+2+i}\right)=3^{-i} w\left(Y^{k+2}\right)
$$

for $i=0, \ldots, n-k-2$. Thus, $w\left(X^{n}\right) \asymp w\left(Y^{n}\right)$ with $C(\asymp)=1 /\left(3 \epsilon_{0}\right)^{2}$.

If $|n-m| \leq 1$, but $n \neq m$, we may assume that $m=n+1$. If $Y^{\prime}$ is the unique $n$-tile that contains $Y$, then by the first part of the proof we have $w(X) \asymp w\left(Y^{\prime}\right) \asymp w(Y)$ with implicit constants independent of the tiles and their levels. The statement follows.

\section{Construction of the geodesic metric}

Based on the concept of weights introduced in the previous section, we can now define a new metric $\varrho$ on our given tree $(\mathbf{T}, d)$. For this purpose we first define a sequence of distance functions $\varrho_{n}$ on $\mathbf{T}$.

Let $n \in \mathbb{N}$ and $x, y \in \mathbf{T}$. Then we define

$$
\begin{aligned}
\varrho_{n}(x, y):=\inf \left\{\operatorname{length}_{w}(P):\right. & P \text { is an } \\
& n \text {-chain joining } x \text { and } y\} .
\end{aligned}
$$

If $x \neq y$, let $P_{x y}^{n}$ be the simple, and $P$ be an arbitrary $n$-chain joining $x$ and $y$. Then we deduce from Lemma 5.2 (ii) that $\operatorname{length}_{w}\left(P_{x y}^{n}\right) \leq$ length $_{w}(P)$. It follows that

$$
\varrho_{n}(x, y)=\operatorname{length}_{w}\left(P_{x y}^{n}\right)
$$

for distinct points $x, y \in \mathbf{T}$.

In this section we will show that the distance functions $\varrho_{n}$ have a limit $\varrho_{n} \rightarrow \varrho$ as $n \rightarrow \infty$, and that $\varrho$ is a geodesic metric on $\mathbf{T}$. We will 
see in the next section that $(\mathbf{T}, d)$ and $(\mathbf{T}, \varrho)$ are quasisymmetrically equivalent. Finally, in Section 9 we will show that by choosing the parameter $\epsilon_{0}$ used in the definition of weights suitably small, we can arrange the Hausdorff dimension of $(\mathbf{T}, \varrho)$ to be arbitrarily close to 1 .

We start with some simple observations.

Lemma 7.1. For each $n \in \mathbb{N}$ the following statements are true:

(i) $\varrho_{n}(x, y)=\varrho_{n}(y, x)$ for $x, y \in \mathbf{T}$.

(ii) $\varrho_{n}(x, y) \leq \varrho_{n}(x, z)+\varrho_{n}(z, y)$ for $x, y, z \in \mathbf{T}$.

This shows that $\varrho_{n}$ is symmetric and satisfies the triangle inequality. However, it is not a metric. Indeed, it is immediate from the definition that

$$
\varrho_{n}(x, x)=\inf \{w(X): X \text { is an } n \text {-tile with } x \in X\}>0
$$

for $x \in \mathbf{T}$.

Proof of Lemma 7.1. (i) Let $x, y \in \mathbf{T}$ be arbitrary. The $n$-tiles $X_{1}, \ldots, X_{r}$ then form an $n$-chain $P$ joining $x$ and $y$ if and only if the $n$-tiles $X_{r}, \ldots, X_{1}$ form an $n$-chain $\widetilde{P}$ joining $y$ and $x$. Moreover, we have $\operatorname{length}_{w}(P)=\operatorname{length}_{w}(\widetilde{P})$. If we take the infimum over all such $P$ here, then (i) follows.

(ii) Let $x, y, z \in \mathbf{T}$ be arbitrary. Suppose that the $n$-tiles $X_{1}, \ldots, X_{r}$ form an $n$-chain $P$ joining $x$ and $z$, and the $n$-tiles $Y_{1}, \ldots, Y_{s}$ form an $n$-chain $\widetilde{P}$ joining $z$ and $y$. Then the $n$-tiles

$$
X_{1}, \ldots, X_{r}, Y_{1}, \ldots, Y_{s}
$$

form an $n$-chain $Q$ joining $x$ and $y$. Note that $X_{r} \cap Y_{1} \neq \emptyset$, because $z \in X_{r} \cap Y_{1}$. We have $\operatorname{length}_{w}(Q)=\operatorname{length}_{w}(P)+\operatorname{length}_{w}(\widetilde{P})$. If we take the infimum over all $P$ and $\widetilde{P}$ here, then (ii) follows.

We now prepare the proof of the convergence of the sequence $\left\{\varrho_{n}\right\}$.

Lemma 7.2. Let $n, k \in \mathbb{N}$ with $k>n$, and $x, y \in \mathbf{T}$ with $x \neq y$ be arbitrary. Then we have

$$
\operatorname{length}_{w}\left(P_{x y}^{k}\right) \leq \operatorname{length}_{w}\left(P_{x y}^{n}\right)+\frac{1}{2} w(X)+\frac{1}{2} w(Y),
$$

where $X$ is the first tile in $P_{x y}^{n}$ and $Y$ the last tile in $P_{x y}^{n}$.

Proof. Let $n \in \mathbb{N}$ and $x, y \in \mathbf{T}$ with $x \neq y$ be arbitrary. Suppose the simple $n$-chain $P=P_{x y}^{n}$ joining $x$ and $y$ is given by the $n$-tiles $X_{1}, \ldots, X_{r}$, where $r \in \mathbb{N}$. Let $X=X_{1}$ be the first tile and $Y=X_{r}$ be the last tile in $P$. Then $x \in X$ and $y \in Y$. For $i=1, \ldots, r-1$ let $v_{i}$ 
be the $n$-vertex where $X_{i}$ and $X_{i+1}$ intersect. We also set $v_{0}:=x$ and $v_{r}:=y$.

For $i=1, \ldots, r$ let $P_{i}$ be the unique simple $(n+1)$-chain joining $v_{i-1}$ and $v_{i}$. Since $\left[v_{i-1}, v_{i}\right] \subset X_{i}$, the chain $P_{i}$ consists of $(n+1)$ tiles contained in $X_{i}$. If we concatenate $P_{1}, \ldots, P_{r}$, then we obtain the simple $(n+1)$-chain $P_{x y}^{n+1}$ joining $x$ and $y$ (see Lemma 5.3 (i)).

By Lemma 6.1 (ii) we have length ${ }_{w}\left(P_{i}\right) \leq w\left(X_{i}\right)$ for $i=2, \ldots, r-1$, because in this case the simple chain $P_{i}$ joins two distinct points in $\partial X_{i}$. We also have length $\left(P_{1}\right) \leq \frac{4}{3} w\left(X_{1}\right)$, and $\operatorname{length}_{w}\left(P_{r}\right) \leq \frac{4}{3} w\left(X_{r}\right)$ by Lemma 6.1 (i). It follows that

$$
\begin{aligned}
\operatorname{length}\left(P_{x y}^{n+1}\right) & =\sum_{i=1}^{r} \operatorname{length}_{w}\left(P_{i}\right) \\
& \leq \sum_{i=1}^{r} w\left(X_{i}\right)+\frac{1}{3} w\left(X_{1}\right)+\frac{1}{3} w\left(X_{r}\right) \\
& =\operatorname{length}_{w}\left(P_{x y}^{n}\right)+\frac{1}{3} w(X)+\frac{1}{3} w(Y) .
\end{aligned}
$$

We now iterate this procedure by increasing the level by 1 in each step until we reach level $k$. In this way, we see that

$$
\operatorname{length}_{w}\left(P_{x y}^{k}\right) \leq \operatorname{length}_{w}\left(P_{x y}^{n}\right)+\frac{1}{3} \sum_{i=n}^{k-1}\left(w\left(X^{i}\right)+w\left(Y^{i}\right)\right),
$$

where $X^{i}$ and $Y^{i}$ are $i$-tiles for $i=n, \ldots, k-1$ with

$$
x \in X^{k-1} \subset \ldots \subset X^{n+1} \subset X^{n}=X
$$

and

$$
y \in Y^{k-1} \subset \ldots \subset Y^{n+1} \subset Y^{n}=Y .
$$

It follows from 6.8 and these inclusions that

$$
w\left(X^{i}\right) \leq 3^{n-i} w(X) \text { and } w\left(Y^{i}\right) \leq 3^{n-i} w(Y)
$$

for $i=n, \ldots, k-1$, and so

$$
\begin{aligned}
\sum_{i=n}^{k-1}\left(w\left(X^{i}\right)+w\left(Y^{i}\right)\right) & \leq(w(X)+w(Y)) \sum_{i=n}^{\infty} 3^{n-i} \\
& \leq \frac{3}{2}(w(X)+w(Y))
\end{aligned}
$$

The statement now follows from (7.4).

Lemma 7.3. For all $x, y \in \mathbf{T}$ the limit

$$
\lim _{n \rightarrow \infty} \varrho_{n}(x, y) \in[0, \infty)
$$

exists. 
Proof. If $n \in \mathbb{N}$ and $X^{n}$ is an $n$-tile, then $w\left(X^{n}\right) \leq 3^{-n}$, as follows from our definition of weights. This implies that if $x=y$, then $0 \leq$ $\varrho_{n}(x, y) \leq 3^{-n}$ and so $\lim _{n \rightarrow \infty} \varrho_{n}(x, y)=0$.

Suppose that $x \neq y$. Then it follows from 7.2 and Lemma 7.2 that

$$
\varrho_{k}(x, y) \leq \varrho_{n}(x, y)+3^{-n}
$$

for $k, n \in \mathbb{N}$ with $k \geq n$. Letting $k \rightarrow \infty$, we see that

$$
\limsup _{k \rightarrow \infty} \varrho_{k}(x, y) \leq \varrho_{n}(x, y)+3^{-n}<\infty
$$

Now letting $n \rightarrow \infty$, we conclude that

$$
\limsup _{n \rightarrow \infty} \varrho_{n}(x, y) \leq \liminf _{n \rightarrow \infty} \varrho_{n}(x, y)
$$

So

$$
\limsup _{n \rightarrow \infty} \varrho_{n}(x, y)=\liminf _{n \rightarrow \infty} \varrho_{n}(x, y)<\infty .
$$

Hence $\lim _{n \rightarrow \infty} \varrho_{n}(x, y)$ exists and is a non-negative (finite) number.

We now define

$$
\varrho(x, y):=\lim _{n \rightarrow \infty} \varrho_{n}(x, y)
$$

for $x, y \in \mathbf{T}$. We know from Lemma 7.1 that $\varrho$ is a non-negative symmetric function that satisfies the triangle inequality. In the proof of Lemma 7.3 we have seen that $\varrho(x, x)=0$ for $x \in \mathbf{T}$.

In order to show that $\varrho$ is a metric on $\mathbf{T}$, it remains to verify that $\varrho(x, y)>0$ whenever $x, y \in \mathbf{T}, x \neq y$. To this end, the following estimates will be useful.

Lemma 7.4. Let $n \in \mathbb{N}$, and $X$ be an $n$-tile. Suppose that $X$ is an arc-tile.

(i) If $p$ and $q$ are the main vertices of $X$, then

$$
\varrho_{k}(p, q)=w(X)
$$

for all $k \geq n$.

(ii) If $u, v \in \partial X$ are two distinct $n$-vertices, then

$$
\epsilon_{0} w(X) \leq \varrho_{k}(u, v) \leq w(X)
$$

for all $k \geq n$.

Proof. (i) Suppose that $p$ and $q$ are the main vertices of $X$. Then the simple $n$-chain $P^{n}$ joining $p$ and $q$ is given by the single tile $X$. Thus $\varrho_{n}(p, q)=\operatorname{length}_{w}\left(P^{n}\right)=w(X)$, and the statement is true for $k=n$. 
Suppose the simple $(n+1)$-chain $P^{n+1}$ joining $p$ and $q$ is given by the $(n+1)$-tiles $X_{1}^{\prime}, \ldots, X_{r}^{\prime}$, where $r \in \mathbb{N}$. Then

$$
\varrho_{n+1}(p, q)=\operatorname{length}_{w}\left(P^{n+1}\right)=\sum_{i=1}^{r} w\left(X_{i}^{\prime}\right)=w(X)
$$

by 6.6 and $(7.2$ ).

For $i=1, \ldots, r-1$ let $v_{i}$ be the $(n+1)$-vertex where $X_{i}^{\prime}$ and $X_{i+1}^{\prime}$ intersect, and set $v_{0}:=p, v_{r}:=q$. Then $v_{i-1}$ and $v_{i}$ are the main vertices of $X_{i}^{\prime}$ for $i=1, \ldots, r$ (see the discussion after (6.6)).

Lemma (i) implies that the simple $(n+2)$-chain $P^{n+2}$ joining $p$ and $q$ is obtained by replacing in $P^{n+1}$ the set $X_{i}^{\prime}$ by the simple $(n+2)$ chain $P_{i}^{n+2}$ joining $v_{i-1}$ and $v_{i}$ for $i=1, \ldots, r$. Since the main vertices of $X_{i}^{\prime}$ are $v_{i-1}$ and $v_{i}$, it follows from 6.6 that length $\left(P_{i}^{n+2}\right)=w\left(X_{i}^{\prime}\right)$. This implies that

$$
\begin{aligned}
\varrho_{n+2}(p, q) & =\operatorname{length}_{w}\left(P^{n+2}\right)=\sum_{i=1}^{r} \operatorname{length}_{w}\left(P_{i}^{n+2}\right)=\sum_{i=1}^{r} w\left(X_{i}^{\prime}\right) \\
& =\operatorname{length}_{w}\left(P^{n+1}\right)=\varrho_{n+1}(p, q)=w(X) .
\end{aligned}
$$

It is clear that we can repeat this argument for higher and higher levels, and (i) follows.

(ii) Suppose $u, v \in \partial X$ are distinct $n$-vertices. Then the desired upper bound follows from a reasoning similar to that in (i) if we use the first part of Lemma 6.1 (ii) instead of (6.6) on each level.

In order to verify the lower bound, we may assume $k \geq n+1$, because $\varrho_{n}(u, v)=w(X)$. Let $P^{n+1}$ be the simple $(n+1)$-chain joining $u$ and $v$ given by the $(n+1)$-tiles $Y_{1}^{\prime}, \ldots, Y_{s}^{\prime}$, where $s \in \mathbb{N}$. We know that $s \geq 3$ by Lemma 5.4 (ii) and our choice of $\delta$. Let $u^{\prime}$ be the $(n+1)$-vertex where $Y_{1}^{\prime}$ and $Y_{2}^{\prime}$ intersect. Then $u^{\prime}$ is the point given by Lemma 5.5. This means $u$ and $u^{\prime}$ are the main vertices of $Y_{1}^{\prime}$ (see the discussion after (6.7)). For each $k \geq n+1$ the simple $k$-chain $P_{u v}^{k}$ joining $u$ and $v$ contains the simple chain $P_{u u^{\prime}}^{k}$ joining $u$ and $u^{\prime}$ as a subchain, which follows from Lemma 5.3 (i), Applying (i) to the tile $Y_{1}^{\prime}$, we see that

$$
\begin{aligned}
\varrho_{k}(u, v) & =\operatorname{length}_{w}\left(P_{u v}^{k}\right) \geq \operatorname{length}_{w}\left(P_{u u^{\prime}}^{k}\right) \\
& =\varrho\left(u, u^{\prime}\right)=w\left(Y_{1}^{\prime}\right) \geq \epsilon_{0} w(X) .
\end{aligned}
$$

This completes the proof of (ii) 
We now introduce a quantity that will allow us to give good estimates for distances of points in $\mathbf{T}$. For distinct $x, y \in \mathbf{T}$ we define

$$
\begin{aligned}
m(x, y):=\max \left\{n \in \mathbb{N}_{0}:\right. & \text { there exist } n \text {-tiles } X \text { and } Y \\
& \text { with } x \in X, y \in Y, \text { and } X \cap Y \neq \emptyset\} .
\end{aligned}
$$

This maximum exists, because by $(5.3)$ for $n$-tiles $X^{n}$ we have

$$
\operatorname{diam}\left(X^{n}\right) \asymp \delta^{n} \rightarrow 0 \text { as } n \rightarrow \infty,
$$

where $d$ is the underlying metric for the diameter of $X^{n}$.

Lemma 7.5. Let $x, y \in \mathbf{T}$ be distinct points, and $m:=m(x, y) \in \mathbb{N}_{0}$. Then

$$
d(x, y) \asymp \delta^{m} \text { and } \varrho(x, y) \asymp w\left(X^{m}\right),
$$

where $X^{m}$ is any $m$-tile containing $x$. Here the implicit constants $C(\asymp)$ are independent of $x$ and $y$.

Proof. By definition of $m$ there exist $m$-tiles $X$ and $Y$ with $x \in X$, $y \in Y$, and $X \cap Y \neq \emptyset$. Then by (5.3),

$$
d(x, y) \leq \operatorname{diam}_{d}(X)+\operatorname{diam}_{d}(Y) \asymp \delta^{m} .
$$

Here the implicit constant is independent of $x$ and $y$.

For the opposite inequality, consider $(m+1)$-tiles $X^{\prime}$ and $Y^{\prime}$ containing $x$ and $y$, respectively. Then $X^{\prime}$ and $Y^{\prime}$ are disjoint by the definition of $m$, and from (5.4) we obtain

$$
d(x, y) \geq \operatorname{dist}_{d}\left(X^{\prime}, Y^{\prime}\right) \geq \delta^{m+1} \asymp \delta^{m} .
$$

Again the implicit constant is independent of $x$ and $y$. The first statement $d(x, y) \asymp \delta^{m}$ follows.

To see the statement for $\varrho$, note that one of the three chains $X$ or $Y$ or $X, Y$ is the simple $m$-chain joining $x$ and $y$. In any case, we have

$$
\begin{aligned}
\varrho(x, y) & =\lim _{k \rightarrow \infty} \varrho_{k}(x, y) \leq \varrho_{m}(x, y)+\frac{1}{2} w(X)+\frac{1}{2} w(Y) \\
& \leq \frac{3}{2}(w(X)+w(Y)) \asymp w(X),
\end{aligned}
$$

as follows from Lemma 7.2 and Lemma 6.2. The latter lemma also implies that the upper bound $\varrho(x, y) \lesssim w(X)$ remains true if we replace $X$ with another $m$-tile containing $x$ (there are at most two such $m$-tiles).

To prove the other inequality, let $X_{1}, \ldots, X_{r}$ with $r \in \mathbb{N}$ be the simple $(m+1)$-chain joining $x$ and $y$. Then $x \in X_{1}$ and $y \in X_{r}$, and so we have $X_{1} \cap X_{r}=\emptyset$ by definition of $m$. Hence $r \geq 3$. Let $u$ be the $(m+1)$-vertex in $X_{1} \cap X_{2}$ and $v$ be the $(m+1)$-vertex in $X_{2} \cap X_{3}$. 
Suppose that $k \geq m+1$, and consider the simple $k$-chain $P_{x y}^{k}$ joining $x$ and $y$. Then $P_{x y}^{k}$ contains the simple $k$-chain $P_{u v}^{k}$ joining $u$ and $v$ as a subchain, and we see that

$$
\begin{aligned}
\varrho_{k}(x, y) & =\operatorname{length}_{w}\left(P_{x y}^{k}\right) \geq \operatorname{length}_{w}\left(P_{u v}^{k}\right)=\varrho_{k}(u, v) \\
& \asymp w\left(X_{2}\right) \asymp w\left(X_{1}\right) \asymp w(X) .
\end{aligned}
$$

Here Lemma 7.4 (ii) and Lemma 6.2 were used. We conclude that

$$
\varrho(x, y)=\lim _{k \rightarrow \infty} \varrho_{k}(x, y) \gtrsim w(X) \text {. }
$$

In the previous inequalities, all implicit constants are independent of $x$ and $y$. The estimate for $\varrho$ follows.

We are now ready for the main result of this section.

Lemma 7.6. The distance function $\varrho$ is a geodesic metric on $\mathbf{T}$.

Proof. Lemma 7.5 immediately implies $\varrho(x, y)>0$ for distinct $x, y \in \mathbf{T}$. This was the last remaining property of a metric we needed to verify for $\varrho$; see the discussion after 7.5). Thus $\varrho$ is a metric on $\mathbf{T}$.

In order to show that $\varrho$ is a geodesic metric, we will establish the following fact.

Claim. $\varrho(x, y)=\varrho(x, z)+\varrho(z, y)$, whenever $x, y \in \mathbf{T}, x \neq y$, and $z \in(x, y)$.

To see this, fix $n \in \mathbb{N}$ and let $P=P_{x y}^{n}$ be the simple $n$-chain joining $x$ and $y$ given by the $n$-tiles $X_{1}, \ldots, X_{r}$, where $r \in \mathbb{N}$. We know that these tiles cover $[x, y]$ (see the proof of Lemma 5.2 (i)), and so there exists a smallest number $1 \leq s \leq r$ such that $z \in X_{s}$. Then $X_{1}, \ldots, X_{s}$ is the simple $n$-chain $Q:=P_{x z}^{n}$ joining $x$ and $z$.

If $z \in X_{s+1}$ (which necessitates $r \geq s+1$ ), then $X_{s+1}, \ldots, X_{r}$ is the simple $n$-chain $Q^{\prime}:=P_{z y}^{n}$ joining $z$ and $y$. Otherwise, if $z \notin X_{s+1}$, this $n$-chain $Q^{\prime}$ is given by $X_{s}, \ldots, X_{r}$. It now follows from $(7.2)$ that

$$
\begin{aligned}
\varrho_{n}(x, z)+\varrho_{n}(z, y) & =\operatorname{length}_{w}(Q)+\operatorname{length}_{w}\left(Q^{\prime}\right) \\
& \leq \sum_{i=1}^{s} w\left(X_{i}\right)+\sum_{i=s}^{r} w\left(X_{i}\right)=\operatorname{length}_{w}(P)+w\left(X_{s}\right) \\
& \leq \varrho_{n}(x, y)+3^{-n} .
\end{aligned}
$$

Letting $n \rightarrow \infty$, we conclude that $\varrho(x, z)+\varrho(z, y) \leq \varrho(x, y)$. Since the opposite inequality is true by the triangle inequality, the Claim follows.

Repeated application of the Claim implies that for all $u, v \in \mathbf{T}$ the length of the arc $[u, v]$ is equal to $\varrho(u, v)$; in other words, $[u, v]$ is a geodesic segment (with respect to the metric $\varrho$ ) joining $u$ and $v$. Hence $\varrho$ is a geodesic metric. 
We summarize the properties of tiles with respect to the metric $\varrho$. Recall that $\mathbf{X}^{n}$ denotes the set of all $n$-tiles.

Proposition 7.7. For all $n, k \in \mathbb{N}_{0}$ the following statements are true:

(i) $\operatorname{diam}_{\varrho}(X) \asymp w(X)$ for all $X \in \mathbf{X}^{n}$.

(ii) $\operatorname{diam}_{\varrho}(X) \asymp \operatorname{diam}_{\varrho}(Y)$ if $|n-k| \leq 1, X \in \mathbf{X}^{n}, Y \in \mathbf{X}^{k}$, and $X \cap Y \neq \emptyset$.

(iii) $\operatorname{diam}_{\varrho}(Y) \lesssim 3^{-k} \operatorname{diam}_{\varrho}(X)$ if $X \in \mathbf{X}^{n}, Y \in \mathbf{X}^{n+k}$, and $X \cap Y \neq$ $\emptyset$.

(iv) $\varrho(x, y) \asymp \operatorname{diam}_{\varrho}\left(X^{m}\right)$ for all distinct $x, y \in \mathbf{T}$, where $m=$ $m(x, y)$ and $X^{m}$ is any $m$-tile with $x \in X^{m}$.

Here the implicit constants are independent of the tiles and their levels in (i) (iii), and independent of $x, y, X^{m}$ in (iv).

Proof. (i) We will actually show that

$$
\epsilon_{0}^{2} w(X) \leq \operatorname{diam}_{\varrho}(X) \leq 2 w(X) .
$$

If $x, y \in X$ with $x \neq y$, then the tile $X$ constitutes $P_{x y}^{n}$, the simple $n$-chain joining $x$ and $y$. So it follows from Lemma 7.2 that for $k \geq n$ we have

$$
\varrho_{k}(x, y)=\operatorname{length}_{w}\left(P_{x y}^{k}\right) \leq \operatorname{length}_{w}\left(P_{x y}^{n}\right)+w(X)=2 w(X) .
$$

Letting $k \rightarrow \infty$, we see that $\varrho(x, y) \leq 2 w(x)$, and so $\operatorname{diam}_{\varrho}(X) \leq$ $2 w(X)$ as desired.

If $X$ is an arc-tile, then it follows from Lemma 7.4 (i) that $\varrho(p, q)=$ $w(X)$ for the two main vertices $p$ and $q$ of $X$. Hence $\operatorname{diam}_{\varrho}(X) \geq w(X)$.

Suppose $X$ is a leaf-tile. Then $n \geq 1$ and $\partial X$ is a singleton set consisting of one $n$-vertex $u$. The unique $(n+1)$-tile $X^{\prime} \subset X$ with $u \in X^{\prime}$ is an arc-tile. By what we have seen, it follows that

$$
\operatorname{diam}_{\varrho}(X) \geq \operatorname{diam}_{\varrho}\left(X^{\prime}\right) \geq w\left(X^{\prime}\right)=\epsilon_{0} w(X) .
$$

Finally, if $n=0$ and $X=X^{0}=\mathbf{T}$, then $X$ contains a 1-tile $X^{\prime}$. Then $X^{\prime}$ is an arc- or a leaf-tile, and from what we have seen, we conclude that

$$
\operatorname{diam}_{\varrho}(X) \geq \operatorname{diam}_{\varrho}\left(X^{\prime}\right) \geq \epsilon_{0} w\left(X^{\prime}\right)=\epsilon_{0}^{2} w(X) .
$$

The statement follows.

(ii) This follows from (i) and Lemma 6.2.

(iii) In the given setup, there is an $n$-tile $Y^{\prime}$ with $Y \subset Y^{\prime}$. Then $Y^{\prime} \cap X \neq \emptyset$. So (i) and (ii) imply that

$$
\operatorname{diam}_{\varrho}(Y) \asymp w(Y) \leq 3^{-k} w\left(Y^{\prime}\right) \asymp 3^{-k} \operatorname{diam}_{\varrho}\left(Y^{\prime}\right) \asymp 3^{-k} \operatorname{diam}_{\varrho}(X),
$$

as desired. 
(iv) This follows from (i) and Lemma 7.5

\section{QUASISYMMETRY}

In this section we complete the proof of Theorem 1.2 by showing that the original metric $d$ on $\mathbf{T}$ is quasisymmetrically equivalent to the geodesic metric $\varrho$ constructed above. For this we require the following fact.

Lemma 8.1. The metric space $(\mathbf{T}, \varrho)$ is doubling.

Proof. Let $x \in \mathbf{T}$ and $s>0$ be arbitrary. It suffices to show that the closed ball $\bar{B}_{\varrho}(x, s)$ can be covered with a controlled number of sets of $\varrho$-diameter $<s / 4$.

To see this, for $k \in \mathbb{N}_{0}$ we define

$$
\begin{aligned}
U^{k}(x)=\{y \in \mathbf{T} & \text { : there exist } k \text {-tiles } X \text { and } Y \\
& \text { with } x \in X, y \in Y, \text { and } X \cap Y \neq \emptyset\} .
\end{aligned}
$$

In other words, $U^{k}(x)$ is the union of all $k$-tiles that meet a $k$-tile containing $x$. Note that

$$
U^{k}(x) \backslash\{x\}=\{y \in \mathbf{T} \backslash\{x\}: m(x, y) \geq k\},
$$

where $m(x, y)$ is defined as in (7.6). Indeed, if $y \in \mathbf{T} \backslash\{x\}$ and $m=$ $m(x, y) \geq k$, then there are non-disjoint $m$-tiles $X^{m}$ and $Y^{m}$ with $x \in$ $X^{m}$ and $y \in Y^{m}$. Then the unique $k$-tiles $X^{k}$ and $Y^{k}$ with $X^{k} \supset X^{m}$ and $Y^{k} \supset Y^{m}$ are non-disjoint and contain $x$ and $y$ respectively. So $y \in U^{k}(x) \backslash\{x\}$. Conversely, if $y \in U^{k}(x) \backslash\{x\}$, then $m(x, y) \geq k$ as follows from the definitions of $U^{k}(x)$ and $m(x, y)$.

We have $U^{0}(x)=\mathbf{T} \supset \bar{B}_{\varrho}(x, s)$. Moreover, Proposition 7.7 (i) implies that $\operatorname{diam}_{\varrho}\left(U^{k}(x)\right) \rightarrow 0$ as $k \rightarrow \infty$. Thus there exists a largest number $n \in \mathbb{N}_{0}$ with $\bar{B}_{\varrho}(x, s) \subset U^{n}(x)$.

By definition of $n$ we know that $\bar{B}_{\varrho}(x, s) \not \subset U^{n+1}(x)$. This means that there is a point $y \in \bar{B}_{\varrho}(x, s) \backslash U^{n+1}(x) \subset U^{n}(x) \backslash U^{n+1}(x)$. Then $\varrho(x, y) \leq s$, and $m(x, y)=n$ as follows from 8.1).

Let $k \in \mathbb{N}_{0}$ and $Y^{n+k}$ be an arbitrary $(n+k)$-tile contained in an $n$-tile $Y^{n} \subset U^{n}(x)$. Then there exists an $n$-tile $X^{n}$ with $x \in X^{n}$ and $X^{n} \cap Y^{n} \neq \emptyset$. Then it follows from Proposition 7.7 (ii) (iv) that

$$
\begin{aligned}
\operatorname{diam}_{\varrho}\left(Y^{n+k}\right) & \lesssim 3^{-k} \operatorname{diam}_{\varrho}\left(Y^{n}\right) \\
& \asymp 3^{-k} \operatorname{diam}_{\varrho}\left(X^{n}\right) \asymp 3^{-k} \varrho(x, y) \leq 3^{-k} s .
\end{aligned}
$$

This estimate implies that we can find $k_{0} \in \mathbb{N}_{0}$ independent of $x$ and $s$ such that $\operatorname{diam}_{\varrho}\left(Y^{n+k_{0}}\right)<s / 4$ for all $\left(n+k_{0}\right)$-tiles $Y^{n+k_{0}}$ contained in any $n$-tile $Y^{n} \subset U^{n}(x)$. 
The point $x$ is contained in at most two $n$-tiles, each of which intersects at most $K n$-tiles, where $K$ is the constant from Lemma 5.7 . Thus $U^{n}(x)$ is a union of at most $2(K+1) n$-tiles. Each of these $n$-tiles contains at most $K^{k_{0}}\left(n+k_{0}\right)$-tiles, and all of these $\left(n+k_{0}\right)$-tiles have $\varrho$-diameter $<s / 4$.

Hence $\bar{B}_{\varrho}(x, s) \subset U^{n}(x)$ can be covered by at most $N^{\prime}:=2(K+1) K^{k_{0}}$ sets of $\varrho$-diameter $<s / 4$. Since $N^{\prime}$ is independent of $x$ and $s$, we conclude that the space $(\mathbf{T}, \varrho)$ is doubling.

Lemma 8.2. The identity map $\mathrm{id}_{\mathbf{T}}:(\mathbf{T}, d) \rightarrow(\mathbf{T}, \varrho)$ is a quasisymmetry.

Proof. Let $x \in \mathbf{T}$ and suppose that $\left\{x_{n}\right\}_{n \in \mathbb{N}}$ is a sequence of points with $x \neq x_{n}$ for $n \in \mathbb{N}$. Then Lemma 7.5 implies that, as $n \rightarrow \infty$, we have $d\left(x, x_{n}\right) \rightarrow 0$ if and only if $m\left(x, x_{n}\right) \rightarrow \infty$ if and only if $\varrho\left(x, x_{n}\right) \rightarrow 0$. This shows that the metrics $d$ and $\varrho$ are topologically equivalent, and

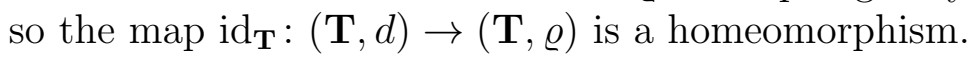

The space $(\mathbf{T}, d)$ is doubling and connected by assumption, and $(\mathbf{T}, \varrho)$ is doubling by Lemma 8.1. So in order to prove that id $\mathbf{T}:(\mathbf{T}, d) \rightarrow$ $(\mathbf{T}, \varrho)$ is a quasisymmetry, it is enough to show that it is a weak quasisymmetry (see [He01, Theorem 10.19]). This means that we have to find a constant $H \geq 1$ such that we have the implication

$$
d(x, y) \leq d(x, z) \Rightarrow \varrho(x, y) \leq H \varrho(x, z)
$$

for all $x, y, z \in \mathbf{T}$.

Let $x, y, z \in \mathbf{T}$ with $d(x, y) \leq d(x, z)$ be arbitrary. We may assume that the points $x, y, z$ are pairwise distinct. Let $m:=m(x, y)$ and $n:=m(x, z)$ be defined as in (7.6).

By the first part of Lemma 7.5 we have $\delta^{m} \asymp d(x, y) \leq d(x, z) \asymp \delta^{n}$. Thus there is a constant $k_{0} \in \mathbb{N}_{0}$ independent of $x, y, z$ such that

$$
n \leq m+k_{0} .
$$

For $i=m, \ldots, m+k_{0}$ we choose an $i$-tile $Y^{i}$ that contains $x$. By applying Proposition 7.7 (ii) at most $k_{0}$ times (and so a number of times independent of $x, y, z)$, we see that

$$
\operatorname{diam}_{\varrho}\left(Y^{m+k_{0}}\right) \asymp \operatorname{diam}_{\varrho}\left(Y^{m}\right) .
$$

We also choose an $n$-tile $Z^{n}$ that contains $x$. Since $m+k_{0} \geq n$, and $x \in Y^{m+k_{0}} \cap Z^{n}$, Proposition 7.7 (iii) implies that

$$
\operatorname{diam}_{\varrho}\left(Y^{m+k_{0}}\right) \lesssim \operatorname{diam}_{\varrho}\left(Z^{n}\right) .
$$

So with Proposition 7.7 (iv) we arrive at

$$
\varrho(x, y) \asymp \operatorname{diam}_{\varrho}\left(Y^{m}\right) \asymp \operatorname{diam}_{\varrho}\left(Y^{m+k_{0}}\right) \lesssim \operatorname{diam}_{\varrho}\left(Z^{n}\right) \asymp \varrho(x, z) .
$$


Since all implicit constants in the previous estimates are independent of $x, y, z$, the statement follows.

The proof of Theorem 1.2 is now complete.

\section{LOWERING THE HAUSdORFF DIMENSION}

In this section we will prove Theorem 1.3 . We assume that $\mathbf{T}$ is the given qc-tree as before. Let $\alpha>1$ be arbitrary. We claim that $\operatorname{dim}_{H}(\mathbf{T}, \varrho) \leq \alpha$ for the Hausdorff dimension of $(\mathbf{T}, \varrho)$ if we choose the parameter $\epsilon_{0}>0$ in (6.2) that was used in the construction of $\varrho$ as described in the previous sections small enough. Then Theorem 1.3 immediately follows, because $T:=(\mathbf{T}, \varrho)$ is a geodesic tree that is quasisymmetrically equivalent to $\mathbf{T}$ and we have $\operatorname{dim}_{H} T \leq \alpha$.

As before, let $K$ be the constant from Lemma 5.7. Then we can choose $\epsilon_{0}>0$ so small (in addition to our previous requirement (6.2) ) that

$$
L:=(1 / 3)^{\alpha-1}+K \epsilon_{0}^{\alpha}<1 .
$$

We claim that with these choices we have $\mathcal{H}^{\alpha}(\mathbf{T}, \varrho)=0$ for the $\alpha$ Hausdorff measure of $(\mathbf{T}, \varrho)$ (we will recall the relevant definitions below). This in turn implies the desired inequality $\operatorname{dim}_{H}(\mathbf{T}, \varrho) \leq \alpha$.

To see that $\mathcal{H}^{\alpha}(\mathbf{T}, \varrho)=0$, we first consider an $n$-tile $X$, where $n \in \mathbb{N}_{0}$. In the following estimates, $X^{\prime}$ denotes an arbitrary $(n+1)$-tile with $X^{\prime} \subset X$ and we denote by $\lambda\left(X^{\prime}\right):=w\left(X^{\prime}\right) / w(X)$ the relative weight of $X^{\prime}$. Note that $\epsilon_{0} \leq \lambda\left(X^{\prime}\right) \leq 1 / 3$ (see $(6.8)$ ).

Suppose first that $X$ is an arc-tile. Let $p$ and $q$ be the main vertices of $X$. Then we have

$$
\sum_{X^{\prime} \cap[p, q] \neq \emptyset} \lambda\left(X^{\prime}\right)=1,
$$

as follows from (6.6). This shows that

$$
\begin{aligned}
\sum_{X^{\prime}} w\left(X^{\prime}\right)^{\alpha} & =w(X)^{\alpha} \sum_{X^{\prime}} \lambda\left(X^{\prime}\right)^{\alpha} \\
& =w(X)^{\alpha}\left(\sum_{X^{\prime} \cap[p, q] \neq \emptyset} \lambda\left(X^{\prime}\right)^{\alpha}+\sum_{X^{\prime} \cap[p, q]=\emptyset} \lambda\left(X^{\prime}\right)^{\alpha}\right) \\
& =w(X)^{\alpha}\left(\sum_{X^{\prime} \cap[p, q] \neq \emptyset} \lambda\left(X^{\prime}\right)^{\alpha-1} \lambda\left(X^{\prime}\right)+\sum_{X^{\prime} \cap[p, q]=\emptyset} \epsilon_{0}^{\alpha}\right) \\
& \leq w(X)^{\alpha}\left((1 / 3)^{\alpha-1}+K \epsilon_{0}^{\alpha}\right)=L w(X)^{\alpha} .
\end{aligned}
$$

For a leaf-tile $X$ or for the 0 -tile $X=X^{0}=\mathbf{T}$ we have

$$
\sum_{X^{\prime}} w\left(X^{\prime}\right)^{\alpha} \leq K \epsilon_{0}^{\alpha} w(X)^{\alpha} \leq L w(X)^{\alpha},
$$


and so we have the same upper bound as for an arc-tile $X$.

Now let $t>0$, and consider

$$
\mathcal{H}_{t}^{\alpha}(\mathbf{T}, \varrho):=\inf \left\{\sum_{i \in \mathbb{N}} \operatorname{diam}_{\varrho}\left(A_{i}\right)^{\alpha}\right\},
$$

where the infimum is taken over all countable covers $\left\{A_{i}\right\}_{i \in \mathbb{N}}$ of $\mathbf{T}$ by sets $A_{i} \subset \mathbf{T}$ with $\operatorname{diam}_{\varrho}\left(A_{i}\right) \leq t$ for $i \in \mathbb{N}$.

We can choose $n \in \mathbb{N}$ large enough so that for each $n$-tile $X$ we have

$$
\operatorname{diam}_{\varrho}(X) \leq 2 w(X) \leq 2 \cdot 3^{-n} \leq t .
$$

Here we used (7.7) in the first inequality. Then

$$
\begin{aligned}
\mathcal{H}_{t}^{\alpha}(\mathbf{T}, \varrho) & \leq \sum_{X \in \mathbf{X}^{n}} \operatorname{diam}_{\varrho}(X)^{\alpha} \leq 2^{\alpha} \sum_{X \in \mathbf{X}^{n}} w(X)^{\alpha} \leq 2^{\alpha} L \sum_{\tilde{X} \in \mathbf{X}^{n-1}} w(\tilde{X})^{\alpha} \\
& \leq \cdots \leq 2^{\alpha} L^{n} w\left(X^{0}\right)^{\alpha}=2^{\alpha} L^{n}
\end{aligned}
$$

where $X^{0}=\mathbf{T}$ is the unique 0 -tile and $w\left(X^{0}\right)=1$. Since $L<1$, and $n \rightarrow \infty$ as $t \rightarrow 0^{+}$, this implies

$$
\mathcal{H}^{\alpha}(\mathbf{T}, \varrho):=\lim _{t \rightarrow 0^{+}} \mathcal{H}_{t}^{\alpha}(\mathbf{T}, \varrho)=2^{\alpha} \lim _{n \rightarrow \infty} L^{n}=0,
$$

as desired. The proof of Theorem 1.3 is now complete.

\section{REMARKS AND OPEN PROBLEMS}

The general strategy to prove the quasisymmetric equivalence of $(\mathbf{T}, d)$ and $(\mathbf{T}, \varrho)$ follows a pattern that has been employed before (for example, see the proof of [BM17, Theorem 18.1]). In the follow-up paper [BM20] we will state general conditions that ensure quasisymmetric equivalence in similar situations. This approach is closely related to recent work by Kigami (see Kig18).

It is an interesting problem whether every qc-tree $\mathbf{T}$ admits a quasisymmetric embedding $\varphi: \mathbf{T} \rightarrow \mathbb{C}$ into the complex plane and whether one can obtain an image $T:=\varphi(\mathbf{T})$ with good geometric properties. For example, one can ask whether for a suitable quasisymmetric embedding $\varphi$ the image $T$ is quasi-convex with respect to the Euclidean metric (then $T$ is geodesic if equipped with its internal path metric) and $\mathbb{C} \backslash T$ is a nice domain (such as a John domain).

For a given tree $\mathbf{T} \subset \mathbb{C}$, we may consider the conformal map $\varphi: \widehat{\mathbb{C}} \backslash$ $\overline{\mathbb{D}} \rightarrow \widehat{\mathbb{C}} \backslash \mathbf{T}$. Here $\widehat{\mathbb{C}}=\mathbb{C} \cup\{\infty\}$ is the Riemann sphere and $\mathbb{D}=\{z \in$ $\mathbb{C}:|z|<1\}$ the unit disk. Since $\mathbf{T}$ is locally connected, $\varphi$ extends to a map on the boundary $f: \partial \mathbb{D} \rightarrow \mathbf{T}$ by Carathéodory's theorem and one obtains an equivalence relation on $\partial \mathbb{D}$ given by $s \sim t \Leftrightarrow f(s)=f(t)$. 
Lin and Rohde have recently studied which equivalence relations $\sim$ arise in this way from trees $\mathbf{T} \subset \mathbb{C}$, where $\widehat{\mathbb{C}} \backslash \mathbf{T}$ is a John domain (see [LR18]). In particular, they were interested in related questions for the continuum random tree (CRT) (see [BT20] for references and relevant facts about the CRT in a related setting). The CRT is geodesic, but not doubling, and so not a qc-tree according to our terminology.

This leads to the question, whether a tree that is of bounded turning, but not necessarily doubling, admits a uniformization similar to the one in Theorem 1.2. In Me11] it is shown that an arc is of bounded turning if and only if it is the image of $[0,1]$ under a weak quasisymmetry. In analogy, one may ask whether a tree that is of bounded turning is the image of a geodesic tree under a weak quasisymmetry.

Trees and tree-like spaces often appear in data structures. Our subdivision procedure as described in Section 5 essentially gives an algorithm to decompose trees with good geometric control. It would be interesting to see whether this procedure has applications in a data-related context.

Acknowledgments. We would like to thank Steffen Rohde for some interesting discussions, in particular about Lemma 3.3. We are also grateful to Guy C. David for reminding us of the reference [Kin17], which we had overlooked in a first version of this paper. Finally, we thank the anonymous referee and the editor Henryk Toruńczyk for various helpful comments.

M.B. was partially supported by NSF grants DMS-1506099 and DMS1808856 .

\section{REFERENCES}

[Az15] J. Azzam, Hausdorff dimension of wiggly metric spaces, Ark. Mat. 53 (2015), $1-36$.

[BiT01] C.J. Bishop and J.T. Tyson, Conformal dimension of the antenna set, Proc. Amer. Math. Soc. 129 (2001), 3631-3636.

[Bo06] M. Bonk, Quasiconformal geometry of fractals, in: Proc. Internat. Congr. Math. (Madrid 2006), Vol. II, Eur. Math. Soc., Zürich, 2006, pp. 1349-1373.

[Bo11] M. Bonk, Uniformization of Sierpinsski carpets in the plane, Invent. Math. 186 (2011), 559-665.

[BM17] M. Bonk and D. Meyer, Expanding Thurston Maps, Amer. Math. Soc., Providence, RI, 2017.

[BM20] M. Bonk and D. Meyer, Uniformly branching trees, in preparation.

[BT20] M. Bonk and H. Tran, The continuum self-similar tree, preprint, 2020, https://arxiv.org/abs/1803.09694.

[CG93] L. Carleson and T.H. Gamelin, Complex Dynamics, Springer, New York, 1993. 
[He01] J. Heinonen, Lectures on Analysis on Metric Spaces, Springer, New York, 2001.

[KK00] M. Kapovich and B. Kleiner, Hyperbolic groups with low-dimensional boundary, Ann. Sci. École Norm. Sup. (4) 33 (2000), 647-669.

[Kig18] J. Kigami, Weighted partition of a compact metrizable space, its hyperbolicity and Ahlfors regular conformal dimension, preprint, 2018, https: //arxiv.org/abs/1806.06558.

[Kin17] K. Kinneberg, Conformal dimension and boundaries of planar domains, Trans. Amer. Math. Soc. 369 (2017), 6511-6536.

[Kul94] W. Kulpa, Sandwich type theorems, Acta Univ. Carolin. Math. Phys. 35 (1994), 45-50.

[Kur68] K. Kuratowski, Topology. Vol. II. Academic Press, New York-London; PWN-Polish Scientific Publishers, Warsaw, 1968.

[LR18] P. Lin and S. Rohde, Conformal welding of dendrites, preprint, 2018.

[MT10] J.M. Mackay and J.T. Tyson, Conformal dimension. Theory and application. University Lecture Series 54. Amer. Math. Soc., Providence, RI, 2010.

[Me11] D. Meyer, Bounded turning circles are weak-quasicircles, Proc. Amer. Math. Soc. 139 (2011), 1751-1761.

[Na92] S.B. Nadler, Jr., Continuum theory. An introduction. Monographs and Textbooks in Pure and Appl. Math., 158. Marcel Dekker, New York, 1992.

[TV80] P. Tukia and J. Väisälä, Quasisymmetric embeddings of metric spaces, Ann. Acad. Sci. Fenn. Ser. A I Math. 5 (1980), 97-114.

[Wh63] G.T. Whyburn, Analytic topology, Colloquium. Publ., Vol. 28, Amer. Math. Soc., Providence, RI, 1963.

Department of Mathematics, University of California, Los Angeles, CA 90095, USA

E-mail address: mbonk@math.ucla.edu

Department of Mathematical Sciences, University of Liverpool, Liverpool L69 7ZL, United Kingdom

E-mail address: dmeyermail@gmail.com 